\title{
FUNCTIONAL MODEL OF A CLOSED NON-SELFADJOINT OPERATOR
}

\author{
VLADIMIR RYZHOV
}

\begin{abstract}
We construct the symmetric functional model for an arbitrary closed operator with a non-empty resolvent set acting on a separable Hilbert space. The main techniques of the study are based on the explicit form of the Sz.-Nagy-Foiaş model for a closed dissipative operator, the Potapov-Ginzburg transform of characteristic functions, and certain resolvent identities. All considerations are carried out under minimal assumptions, and obtained results are directly applicable to problems typically arising in mathematical physics. Explicit formulae for all objects relevant to the model construction are provided.
\end{abstract}

\section{INTRODUCTION}

The functional model of non-selfadjoint dissipative operators plays a prominent role in applications of operator theory in mathematical physics. The theory was initiated, developed and further elaborated in numerous works, see books $[10,12$, $14,24,32,33,34]$ and references therein. Today it is essentially complete and is commonly known as the model associated with names of B. Sz.-Nagy and C. Foiaş, L. de Branges and J. Rovnyak, P. Lax and R. Phillips.

An application-motivated perspective on the functional model for dissipative operators was developed in (and greatly influenced by) works of B. Pavlov, see [35, 36], whose ideas are deeply rooted in the scattering theory of P. Lax and R. Philips [24]. The hallmark of the latter theory is its renowned clearness and transparency of underlaying ideas. As a result of this influence, B. Pavlov's form of functional model, later coined as the symmetric functional model, provided a convenient and intuitively coherent framework for study of various problems in mathematical physics associated with dissipative operators. Here we only mention the dissipative Schrödinger operator with complex potential $[35,36]$ and with non-selfadjoint boundary condition [37], the Boltzmann operator [23, 30], and stochastic quantum dynamics [39], see survey [38] for more details and examples. This orientation toward practical aspects is not something accidental; the symmetric model emerged as an extension of well-known methods of classical spectral analysis developed within the "selfadjoint" theory commonly used in applications [40]. The model construction is based on expansions by canonical systems of eigenfunctions of the absolutely continuous spectrum of a certain selfadjoint operator (the dilation) followed by the mapping of the Hilbert space where the initial dissipative operator acts into the model space. Clear physical meaning of all objects participating in the theory

Date: February 2, 2007.

1991 Mathematics Subject Classification. 47A20, 47A45, 47A48.

Key words and phrases. functional models, non-selfadjoint operators, non-dissipative operators, Potapov-Ginzburg transform. 
combined with its intuitive appeal inherited from the Lax-Philips theory greatly contributed to the success of symmetric functional model as an adequate tool for the study of various problems of mathematical physics. (See papers $[1,2,3]$ for the application of symmetric model technique in a few concrete situations and $[8,9,17]$ for recent applications in the semiconductor theory.)

After the distinct success of the functional model for dissipative operators and contractions was realized, the question of building a model for an operator with the dissipativity condition omitted raised naturally. Owing to the properties of Cayley transform, this problem is formally equivalent to the model construction for a non-contractive bounded operator. In the course of conducted research it became clear that the contractiveness condition is an essential requirement and can not be relaxed easily. Nevertheless, a few models of certain classes of noncontractive and non-dissipative operators, have been offered in a series of papers $[7,11,13,18,48,49,50,51,52]$. Despite their significant contributions to the abstract operator theory, a certain inconvenience of Cayley transforms for the study of, say the nonselfadjoint Schrödinger operator with complex potential, limits applicability of these models for non-contractions to typical non-dissipative problems arising in mathematical physics. A suitable approach to the spectral analysis of nonselfadjoint non-dissipative operators that simultaneously offers the functional model, was suggested and further developed by S. Naboko in [26, 27, 28]. Apart from the model considerations realized for some class of additive perturbations of a selfadjoint operator, his works significantly contributed to the development of various chapters of the nonselfadjoint operator theory. The relevant results include a highly successful definition of absolutely continuous and singular subspaces, a sophisticated analysis of singular spectra, the functional model for rank one singular perturbations of nonselfadjoint operators, the scattering theory, and some others, see $[20,21,22,29,31]$ in addition to the papers cited above.

Two important features of the model $[26,27,28]$ that distinguish it from its alternatives have to be emphasized; they are its explicitness and its constructiveness. By explicitness we mean the fact that all relevant objects involved in the theory are given in their explicit and final form. For instance, exact formulae are provided for the selfadjoint dilation of a dissipative operator, for the isometry that maps the dilation to the model space, and for the dense set where this isometry is originally defined. Secondly, all calculations and proofs are carried out constructively, with a minimal resorting to general and abstract theorems. In fact, having in hand a non-selfadjoint non-dissipative additive perturbation of a given selfadjoint operator and following the guidance of $[26,27,28]$, one can construct the symmetric model "from scratch", without extensive preliminary knowledge on the theory of non-selfadjoint operators. In addition, all the building blocks of the method are represented straightforwardly in terms of the original problem, namely resolvents of the participating operators and require just a few explicitly defined auxiliaries. These factors make the model theory of $[26,27,28]$ more appealing from the applications perspective than its possible alternatives mentioned earlier.

The key idea behind reasoning of $[26,27,28]$ is essentially perturbative in a sense that it relies on certain resolvent identities typically found in the perturbation theory. The main object of study is a nonselfadjoint operator

$$
L:=A+i V
$$


acting in the Hilbert space $H$, where $A=A^{*}$ and $V=V^{*}$ is $A$-bounded with the relative bound smaller than 1 , so that domains of $A$ and $L$ coincide and operator $L$ is closed. If $V \geq 0$, then $L$ is dissipative and the regular model technique for dissipative operators is applicable. However, if $V=V^{*}$ is not of a definite sign, then an auxiliary operator is needed. It is introduced by the formula

$$
L^{\prime \prime}:=A+i|V|=A+i \frac{\alpha^{2}}{2}
$$

where $\alpha:=\sqrt{2|V|}$ is defined in accordance with the functional calculus for the selfadjoint operator $|V|$. Obviously, $L^{\|}$is dissipative and its symmetric model can be built using the conventional technique. It has to be noted at this point that the work [28] contains an explicit model construction for dissipative operators of the form (1.2) that serves as a necessary preliminary step toward the model for $L$. The operator $L$ can be effectively described in terms of the symmetric model for $L^{11}$ largely owing to this explicitness. Moreover, any operator in the form

$$
L^{\varkappa}:=A+\frac{\alpha \varkappa \alpha}{2}
$$

where the bounded mapping $\varkappa$ satisfies certain additional conditions on its norm, can be represented similarly in the model space of $L^{\|}$, see [28, 29]. Obviously, the family $L^{\varkappa}$ includes operator $L$ as a special case corresponding to the choice $\varkappa=i J$ with $J=\operatorname{sign}(V)$ being an involution $\left(J=J^{*}=J^{-1}\right)$ in the space $E:=\overline{\alpha H}$. There is a remarkable observation made in [26] regarding the so-called characteristic operator-functions of $L$ and $L^{\|}$. It is shown that they are related to each other via the Potapov-Ginbzburg transform known in the theory of spaces with indefinite metric [6]. This circumstance does not play any particular role in argumentation of [26] and is merely a curious by-product of the explicit model construction. In contrast, the Potapov-Ginzburg transform is a key component in considerations below.

Shortly after pioneering works of S. Naboko, N. Makarov and V. Vasyunin exploited the same perturbative idea in their treatment of the case of an arbitrary bounded operator and derived formulae of its action in the model space of a certain contraction [25]. Simultaneously, the question of model construction for an arbitrary closed operator with non-empty resolvent set was solved simply by the virtue of Cayley transform, although on the abstract level. From the applications perspective, the generic answer given by [25] is not quite satisfactory. In particular, it does not offer much help for the model representation in situations like nonselfadjoint extensions of concrete symmetric operators [44], or in similar cases where the operator is not easily representable as a sum of its real and imaginary parts as required by (1.1). Slightly more general settings, such as operators of typical boundary value problems recently studied on the abstract level in [45], are not covered by arguments given in [28,44], and the results of [25] are not easily translatable into the problem's terms either. The paper [48] attempts to deal with these issues by methods of linear relations in Hilbert space. The considerations are carried out on the foundation of Sz.Nagy-Foiaş model for contractions and their relevance to problems of mathematical physics is not quite obvious.

These inconveniences motivate an independent model construction for an arbitrary closed operator with nonempty resolvent set that would serve as a direct generalization of the symmetric model from [28]. The paper offers such a model, free of limitations described above. It was already successfully applied (without a 
proof) to the study of nondissipative operators from a fairly wide class in [42, 43], where the notion of local absolutely continuous and singular subspaces was examined and utilized for the subsequent study of scattering theory for a pair of nonselfadjoint operators. Owing to the generic form of operator under consideration, results obtained in the current paper cover both cases of the model for nonselfadjoint additive perturbations [28] and for extensions of symmetric operators [44]. As a direct consequence, considerations below can be used for study of selfadjoint operators subject to a nonselfadjoint additive perturbations in combination with nonselfadjoint boundary conditions. The model is applicable in more complicated settings, such as the case when the "nonselfadjointness" is caused by a non-additive perturbation of the principal symbol of a (pseudo)differential operator. This situation is found in physics of dissipative and active media and can be viewed as a non-conservative perturbation of the metric induced by the original Hilbert space structure. Since the assumption of dissipativity is omitted, the theory of magnetohydrodynamic instabilities [15] is another possible area where the model given below can be utilized.

The construction offered in the paper is carried out in full accordance with the approach of [28]. It is applicable for an arbitrary closed operator $L$ with nonempty resolvent set acting in a separable Hilbert space. As a preliminary step, we construct the Sz.-Nagy-Foiaş model in its symmetric form for an unbounded dissipative operator and then obtain explicit expressions for the isometry that maps the original Hilbert space into the model one. The availability of explicit formulae for the model mapping is the first key component of the schema from [28]. The next step consists of the solution to the problem delineated above, namely the choice of a "close" dissipative operator that would play the role of $L^{\prime \prime}$ for $L$, the initial nondissipative operator. Since the imaginary part of $L$ is not assumed available, we have to take a detour. The crucial role here is played by the Potapov-Ginzburg transform applied to the characteristic function of $L$. Vaguely speaking, the characteristic function of $L$ is an analytic operator function that possesses certain metric properties with regard to the indefinite metric induced by the nonselfadjoint part of $L$. Its Potapov-Ginzburg transform turns out to be an analytic operator function that coincides with the characteristic function of a certain dissipative operator that is taken then as the "close" operator $L "$. The most important ingredient of all pertinent calculations is the fundamental result of D. Arov [5] cited in Theorem 3.6. On the language of system theory, this result states that the Potapov-Ginzburg transform applied to linear passive dynamic systems maps scattering systems into transmission ones, and in addition, the transfer functions of these systems are related to each other via the Potapov-Ginzburg transform as well. Moreover, this mapping is fully invertible.

Since this result plays such an important role in our argumentation, let us elaborate a bit more on this topic using a slightly simplified setting of a single Hilbert space for the illustration. The exposition we are about to give is lacking proofs; however, all the details can be recovered either by direct calculations or by referring to the relevant literature $[5,6]$. Let $H, E$ be Hilbert spaces and the block operator $\mathcal{A}:=\left(\begin{array}{cc}T & F \\ G & S\end{array}\right)$ be an unitary from $E \oplus H$ into itself. As a direct consequence of equalities $\mathcal{A} \mathcal{A}^{*}=\mathcal{A}^{*} \mathcal{A}=I$, the operator-function $\mathcal{A}(z):=T+z F(I-z S)^{-1} G$ 
with values in the algebra of bounded operators on $E$ is analytic and contractive for $|z|<1$. In the system theory, where operator $\mathcal{A}$ is associated with the so-called scattering linear passive dynamic system, the function $\mathcal{A}(z)$ is identified with the transfer function of system $\mathcal{A}$. Assume the space $E$ is split into two complementary subspaces $E=E_{+} \oplus E_{-}$and $X^{ \pm}$are two orthogonal projections onto $E_{ \pm}$respectively. Projections $X^{ \pm}$turn the space $E$ into the space with indefinite metric $J[\cdot, \cdot]$ defined as $J[x, y]:=(J x, y)_{E}$, where $(x, y)_{E}$ is the Hilbert product of $x, y \in E$, and $J:=X^{+}-X^{-}$. Operator $J$ possesses properties of an involution, that is, it is unitary and selfadjoint at the same time, $J=J^{*}=J^{-1}$. Spaces with indefinite metric induced by such operators called $J$-spaces or Krein spaces [6]. Operator $\mathcal{B}:=\left(\mathcal{X}^{-}+\mathcal{X}^{+} \mathcal{A}\right)\left(\mathcal{X}^{+}+\mathcal{X}^{-} \mathcal{A}\right)^{-1}$, where $\mathcal{X}^{ \pm}:=X^{ \pm} \oplus I_{H}$ are orthogonal projections in $E \oplus H$, is a bounded map of $E \oplus H$ into itself. If we define an involution $\mathcal{J}$ on the orthogonal sum $E \oplus H$ by the formula $\mathcal{J}:=\mathcal{X}^{+}-\mathcal{X}^{-}$, then $\mathcal{B}$ is $\mathcal{J}$-unitary operator. It means the simultaneous fulfillment of two equalities, $\mathcal{J}-\mathcal{B}^{*} \mathcal{J} \mathcal{B}=0$ and $\mathcal{J}-\mathcal{B} \mathcal{J B}^{*}=0$. Blocks of operator $\mathcal{B}$ are bounded operators and they define an operator valued function $\mathcal{B}(z)$ constructed in the same manner as the function $A(z)$ is constructed from the blocks of operator $\mathcal{A}$. The function $\mathcal{B}(z)$ is analytic in some neighborhood $\mathcal{O}$ of the origin. The important property of $\mathcal{B}(z)$, $z \in \mathcal{O}$ is its contractiveness in the indefinite metric $J[\cdot, \cdot]$ on the space $E$. It means the operator inequalities $J-\mathcal{B}(z) J[\mathcal{B}(z)]^{*} \geq 0$ and $J-[\mathcal{B}(z)]^{*} J \mathcal{B}(z) \geq 0$ hold true for $z \in \mathcal{O}$. It turns out that the function $\mathcal{B}(z)$ for $z \in \mathcal{O}$ can be represented in the form $B(z)=\left(X^{-}+X^{+} A(z)\right)\left(X^{+}+X^{-} A(z)\right)^{-1}$, which is a direct analogue of the mapping $\mathcal{A} \mapsto \mathcal{B}$ introduced above. This mapping between $\mathcal{A}$ and $\mathcal{B}$, or equivalently, between $\mathcal{A}(z)$ and $\mathcal{B}(z)$, is called the Potapov-Ginsburg transform (PG-transform). It is fully invertible, meaning that there exists an inversion formula that recovers the operator $\mathcal{A}$ from its $\mathrm{PG}$-transform $\mathcal{B}$, provided the latter is an unitary in the indefinite metric. As one can expect, the function $\mathcal{A}(z)$ is recovered from $\mathcal{B}(z)$ by the same inversion formula. Speaking of systems, the function $\mathcal{B}(z)$ is the transfer function of the linear passive system associated with the operator $\mathcal{B}$, the transmission system. The PG-transform maps the system $\mathcal{A}$ into the systesm $\mathcal{B}$ by redistribution of the external data of $\mathcal{A}$ (i. e. inputs and outputs) according to the decomposition of the external space $E$ into the orthogonal sum $E=E_{+} \oplus E_{-}$.

In application to the problem of building the symmetric model for a non-dissipative operator, the results of the previous paragraph help us to verify the uniqueness of the dissipative operator that we take as "close" in the sense indicated above, and make sure it satisfies our needs. Moreover, relations obtained in course of Potapov-Ginsburg transforms calculations reveal important identities between resolvents of the "close" dissipative operator and operator $L$, which comprise the third cornerstone of the model construction.

The paper consists of this Introduction and three more sections. Section 2 is dedicated to the explicit procedure of passing from a closed dissipative operator (denoted $A$ in the text) to its symmetric functional model. First, employing results of [19] we obtain the selfadjoint dilation $\mathscr{A}$ of operator $A$ and then define the isometry $\Phi$ that realizes $\mathscr{A}$ as a multiplication operator in its spectral representation, the model space. Two other mappings closely related to $\Phi$ are introduced. Their essential parts are expressed via resolvents of $A$ and its adjoint $A^{*}$. Owing to this very fact the resolvent of $L$ be can be effectively described in the model space of $A$. 
Section 3 is a study of the nondissipative operator $L$ from the abstract point of view. By the Cayley transform we reduce the setting to that of bounded operators, thereby making results of linear systems theory [5] directly applicable to our case. With the help of Potapov-Ginzburg transform and arguments from [5] we obtain the explicit form of a certain contractive operator. It is proven then that its Cayley transform is some dissipative operator, which is subsequently taken as "close" to $L$. The section concludes with relations for resolvents of $A$ and $L$ needed for the model construction.

Finally, Section 4 is the Model Theorem and its proof.

Computations required for the paper's purposes are quite extensive, and on a few occasions we permit ourselves to skip some details. However, efforts were made in order to clarify the argumentation and to provide the reader with references to the literature where analogous calculations can be found. Two main resources in this regard are the original work [28] and the recent paper [44] where all relevant computations are supplied in full length. For properties of Potapov-Ginzburg transform and the concise account of results from [5] we refer the reader to the book [6].

The author would like to express his gratitude to Prof. S. Naboko for his interest to the work and continual encouragement.

Notation. Let us recall a few basic facts from the operator theory and agree upon the notation. For two separable Hilbert spaces $H_{1}$ and $H_{2}$ the $\operatorname{sign} A: H_{1} \rightarrow H_{2}$ is used to denote a bounded linear operator $A$ defined everywhere in $H_{1}$ with the range in the space $H_{2}$. Symbols $\mathbb{R}, \mathbb{C}, \mathfrak{I m}(z)$ stand for the real axis, the complex plane, and the imaginary part of a complex number $z \in \mathbb{C}$, respectively. The upper and lower half planes are the open sets $\mathbb{C}_{ \pm}:=\{z \in \mathbb{C} \mid \pm \mathfrak{I m}(z)>0\}$. Similarly, $\mathbb{R}_{ \pm}:=\{k \in \mathbb{R} \mid \pm k>0\}$ and $\mathbb{D}:=\{z \in \mathbb{C}|| z \mid<1\}$. If $A$ is a closed linear operator $A$ on a separable Hilbert space $H$, the domain, range and null space of $A$ are denoted $\operatorname{Dom}(A), \operatorname{Ran}(A)$, and $\operatorname{Ker}(A)$, respectively. The symbol $\rho(A)$ is used for the resolvent set of $A$. We always assume that the domain of a closed operator $A$ is dense in $H$. A sesquilinear form $\Psi_{A}(\cdot, \cdot)$ defined on the product $\operatorname{Dom}(A) \times \operatorname{Dom}(A)$ :

$$
\left.\Psi_{A}(f, g)=\frac{1}{i}[A f, g)_{H}-(f, A g)_{H}\right], \quad f, g \in \operatorname{Dom}(A)
$$

is a substitute for the imaginary part of $A$ in the sense that $2 \mathfrak{I m}(A f, f)=\Psi_{A}(f, f)$, $f \in \operatorname{Dom}(A)$.

Definition 1.1. Operator $A$ is called dissipative if

$$
\mathfrak{I m}(A f, f) \geq 0, \quad f \in \operatorname{Dom}(A),
$$

where $\mathfrak{I m}(\cdot)$ denotes the imaginary part.

Definition 1.2. Operator $A$ is called maximal dissipative if (1.4) holds and the resolvent $(A-z I)^{-1}: H \rightarrow H$ exists for any $z \in \mathbb{C}_{-}$.

There exists a one-to-one correspondence between all closed operators $A$ such that $-i \in \rho(A)$ and bounded operators $T$, defined everywhere on the space $H$ with the property $\overline{\operatorname{Ran}(T-I)}=H$. This correspondence is established by the Cayley transform

$$
A \mapsto T:=(A-i I)(A+i I)^{-1}, \quad T \mapsto A:=(-i)(T+I)(T-I)^{-1}
$$


A brief calculation show that the dissipativity of $A$ is equivalent to the norm estimate $\|T\| \leq 1$, that is, the Cayley transform of $A$ is a contraction. For two separable Hilbert spaces we denote $\mathscr{C}\left(H_{1}, H_{2}\right)$ the set of all densely defined closed operators $A$ with the domain $\operatorname{Dom} A \subset H_{1}$ and the range $\operatorname{Ran}(A) \subset H_{2}$. The symbol $\mathscr{C}^{+}(H)$ is used for the subset of $\mathscr{C}(H, H)$ that consists of all maximal dissipative operators.

\section{Sz.-NAGY-Foiaş MOdEL OF A DISSIPATIVE OPERATOR}

The model construction for a dissipative operator $A$ offered in this Section follows the plan of [36]. It is based on the exact form of the selfadjoint dilation of $A$ and the subsequent passage into its spectral representation. Below we use the form of selfadjoint dilation of a closed dissipative operator found in [19] and follow the schema of [28] where the boundary values of resolvent are used to pass to its spectral representation. We exposition begins with the brief recollection of properties of dissipative operators, definition of their characteristic functions, and continues with some results concerning a certain linear set in the dilation space. After additional considerations regarding its density, which is equivalent to the completeness of two canonical systems of eigenvectors of the dilation, we formulate the model theorem and conclude the Section with a few notes regarding its proof.

2.1. Preliminaries. Let us remind basic definitions and properties of the selfadjoint dilation and the characteristic function of a closed maximal dissipative operator $A$ acting on the Hilbert space $H$.

2.1.1. Boundary operators. Let $T:=(A-i I)(A+i I)^{-1}: H \rightarrow H$ be the Cayley transform (1.5) of $A$. Then $T$ is a contraction, $\|T\| \leq 1$. Denote $R:=i(A+i I)^{-1}$. Following equalities for $T$ and $R$ are easy to verify

$$
\begin{aligned}
& I-T^{*} T=2\left(R+R^{*}-2 R^{*} R\right) \\
& I-T T^{*}=2\left(R+R^{*}-2 R R^{*}\right)
\end{aligned}
$$

Further, since $\|T\| \leq 1$, operators (2.1) are non-negative and the square roots

$$
\Delta_{T}:=\left(I-T^{*} T\right)^{1 / 2}, \quad \Delta_{T^{*}}:=\left(I-T T^{*}\right)^{1 / 2}
$$

are well defined by the functional calculus of selfadjoint operators. Maps (2.1) are called defect operators and closures of their ranges in $H$, the subspaces

$$
\mathcal{E}:=\operatorname{clos} \operatorname{Ran}\left(\Delta_{T}\right), \quad \mathcal{E}_{*}:=\operatorname{clos} \operatorname{Ran}\left(\Delta_{T^{*}}\right),
$$

are called the defect subspaces of contraction $T$ (cf. [32]). The intertwining property $T \Delta_{T}=\Delta_{T^{*}} T$ shows that $T: \mathcal{E} \rightarrow \mathcal{E}_{*}, T^{*}: \mathcal{E}_{*} \rightarrow \mathcal{E}$. Denote $Q:=\frac{1}{\sqrt{2}} \Delta_{T}$, $Q_{*}:=\frac{1}{\sqrt{2}} \Delta_{T^{*}}$ and let $\Gamma \in \mathscr{C}(H, \mathcal{E}), \Gamma_{*} \in \mathscr{C}\left(H, \mathcal{E}_{*}\right)$ be two closed operators defined as closures of mappings $\widetilde{\Gamma}: u \mapsto Q(A+i I) u, \widetilde{\Gamma}_{*}: v \mapsto Q_{*}\left(A^{*}-i I\right) u$, where $u \in \operatorname{Dom}(A), v \in \operatorname{Dom}\left(A^{*}\right)$, correspondingly. The closability of $\widetilde{\Gamma}, \widetilde{\Gamma}_{*}$ follows from the closedness of $A$ and $A^{*}$. Mappings $\Gamma$ and $\Gamma_{*}$ are conventionally termed the boundary operators of $A$ and $-A^{*}$, see [47]. The essence of definition is clarified by the following lemma.

Lemma 2.1. For any $u, v \in \operatorname{Dom}(A), u^{\prime}, v^{\prime} \in \operatorname{Dom}\left(A^{*}\right)$

$$
\Psi_{A}[u, v]=(\Gamma u, \Gamma v)_{\mathcal{E}}, \quad \Psi_{\left(-A^{*}\right)}\left[u^{\prime}, v^{\prime}\right]=\left(\Gamma_{*} u^{\prime}, \Gamma_{*} v^{\prime}\right)_{\mathcal{E}_{*}},
$$

where the form $\Psi_{A}[\cdot, \cdot]$ is defined in (1.3) 
Proof. Let us verify the first identity. To that end note that any vector $u \in \operatorname{Dom}(A)$ is uniquely represented in the form $u=i(A+i I)^{-1} \varphi=R \varphi$ with $\varphi \in H$ and analogously $v=i(A+i I)^{-1} \psi$ with $\psi \in H$ for $v \in \operatorname{Dom}(A)$. Therefore, we have $A R=i A(A+i I)^{-1}=i(I-R)$, hence

$$
\begin{aligned}
\Psi_{A}[u, v] & =(-i)[(A u, v)-(u, A v)]=(-i)[(A R \varphi, R \psi)-(R \varphi, A R \psi)] \\
& =((I-R) \varphi, R \psi)+(R \varphi,(I-R) \psi)=\left(\left(R+R^{*}-2 R^{*} R\right) \varphi, \psi\right) \\
& =(1 / 2)\left(\left(I-T T^{*}\right) \varphi, \psi\right)=(1 / 2)\left(\Delta_{T} \varphi, \Delta_{T} \psi\right)=(Q \varphi, Q \psi) \\
& =\left(Q(A+i I)^{-1} u, Q(A+i I)^{-1} v\right)=(\widetilde{\Gamma} u, \widetilde{\Gamma} v)=(\Gamma u, \Gamma v) .
\end{aligned}
$$

The second formula is proved analogously.

\subsubsection{Selfadjoint dilation and related questions.}

Definition $2.2([32])$. The selfadjoint operator $\mathscr{A}$ on the Hilbert space $\mathscr{H} \supset H$ is called a selfadjoint dilation of $A \in \mathscr{C}^{+}(H)$ if

$$
(A-z I)^{-1}=\left.P_{H}(\mathscr{A}-z I)^{-1}\right|_{H} \quad \text { for any } z \in \mathbb{C}_{-},
$$

where $P_{H}$ denotes the orthogonal projection in $\mathscr{H}$ onto the subspace $H$. The dilation $\mathscr{A}$ is called minimal if

$$
\mathscr{H}=\text { clos } \operatorname{span}\left\{(\mathscr{A}-z I)^{-1} H \mid z \notin \mathbb{R}\right\}
$$

Any operator from $\mathscr{C}^{+}(H)$ has the minimal selfadjoint dilation. Its form below was pointed out in [36] and generalized in the work [19].

Let $\mathscr{D}_{+}:=L_{2}\left(\mathbb{R}_{+}, \mathcal{E}\right), \mathscr{D}_{-}:=L_{2}\left(\mathbb{R}_{-}, \mathcal{E}_{*}\right)$ be the Hilbert spaces of square integrable $\mathcal{E}$ - and $\mathcal{E}_{*}$-valued vector-functions defined on $\mathbb{R}_{ \pm}$. The dilation space is chosen to be the direct sum $\mathscr{H}:=\mathscr{D}_{-} \oplus H \oplus \mathscr{D}_{+}$with elements in the form $\left(h_{-}, h_{0}, h_{+}\right)$, $h_{0} \in H, h_{ \pm} \in \mathscr{D}_{ \pm}$. Define two operators, $\mathscr{A}^{\prime}$ and $\mathscr{A}^{\prime \prime}$ in $\mathscr{H}$, by formulae

$$
\begin{aligned}
& \left\{\begin{aligned}
\operatorname{Dom}\left(\mathscr{A}^{\prime}\right):=\left\{h=\left(h_{-}, h_{0}, h+\right) \in \mathscr{H} \mid\right. \\
\text { 1. } h_{-} \in W_{2}^{1}\left(\mathbb{R}_{-}, \mathcal{E}_{*}\right), \quad h_{+} \in W_{2}^{1}\left(\mathbb{R}_{+}, \mathcal{E}\right) \\
\text { 2. } \quad \varphi:=h_{0}+Q_{*} h_{-}(0) \in \operatorname{Dom}(A) \\
\text { 3. } \left.h_{+}(0)=T^{*} h_{-}(0)+i \Gamma \varphi\right\} \\
\mathscr{A}^{\prime}\left(\begin{array}{l}
h_{-} \\
h_{0} \\
h_{+}
\end{array}\right):=\left(\begin{array}{l}
i \frac{d}{d \xi} h_{-} \\
-i h_{0}+(A+i I) \varphi \\
i \frac{d}{d \xi} h_{+}
\end{array}\right), \quad\left(\begin{array}{l}
h_{-} \\
h_{0} \\
h_{+}
\end{array}\right) \in \operatorname{Dom}\left(\mathscr{A}^{\prime}\right)
\end{aligned}\right. \\
& \operatorname{Dom}\left(\mathscr{A}^{\prime \prime}\right):=\left\{h=\left(h_{-}, h_{0}, h+\right) \in \mathscr{H} \mid\right. \\
& \text { 1. } h_{-} \in W_{2}^{1}\left(\mathbb{R}_{-}, \mathcal{E}_{*}\right), \quad h_{+} \in W_{2}^{1}\left(\mathbb{R}_{+}, \mathcal{E}\right) \\
& \text { 2. } \psi:=h_{0}+Q h_{+}(0) \in \operatorname{Dom}\left(A^{*}\right) \\
& \text { 3. } \left.h_{-}(0)=T h_{+}(0)-i \Gamma_{*} \psi\right\} \\
& \mathscr{A}^{\prime \prime}\left(\begin{array}{l}
h_{-} \\
h_{0} \\
h_{+}
\end{array}\right):=\left(\begin{array}{l}
i \frac{d}{d \xi} h_{-} \\
i h_{0}+\left(A^{*}-i I\right) \psi \\
i \frac{d}{d \xi} h_{+}
\end{array}\right), \quad\left(\begin{array}{l}
h_{-} \\
h_{0} \\
h_{+}
\end{array}\right) \in \operatorname{Dom}\left(\mathscr{A}^{\prime \prime}\right)
\end{aligned}
$$


where $W_{2}^{1}(\cdot, \cdot)$ are vector-valued Sobolev classes and $\xi \in \mathbb{R}_{ \pm}$are the arguments of functions from $\mathscr{D}_{ \pm}$. Existence of boundary values $h_{ \pm}(0)$ follows from the Sobolev imbedding theorems.

Theorem 2.3. Operators $\mathscr{A}^{\prime}$ and $\mathscr{A}^{\prime \prime}$ coincide and $\mathscr{A}:=\mathscr{A}^{\prime}=\mathscr{A}^{\prime \prime}$ is the selfadjoint dilation of $A$.

Proof. Results of the paper [19] show that $\mathscr{A}^{\prime}$ is the selfadjoint dilation of $A$. Let us prove the equality $\mathscr{A}^{\prime}=\mathscr{A}^{\prime \prime}$. Assume $\left(h_{-}, h_{0}, h_{+}\right) \in \operatorname{Dom}\left(\mathscr{A}^{\prime}\right)$. Then, using notation introduced in (2.3) and (2.4),

$$
\begin{aligned}
\psi & =h_{0}+Q h_{+}(0)=h_{0}+Q\left[T^{*} h_{-}(0)+i \Gamma \varphi\right] \\
& =h_{0}+T^{*} Q_{*} h_{-}(0)+i Q^{2}(A+i I) \varphi \\
& =h_{0}+T^{*} Q_{*} h_{-}(0)+i\left(R+R^{*}-R^{*} R\right)(A+i I) \varphi \\
& =h_{0}+T^{*} Q_{*} h_{-}(0)-\left[I+2 i\left(A^{*}-i I\right)^{-1}\right] \varphi+\left(A^{*}-i I\right)^{-1}(A+i I) \varphi \\
& =h_{0}+T^{*}\left(Q_{*} h_{-}(0)-\varphi\right)+\left(A^{*}-i I\right)^{-1}(A+i I) \varphi \\
& =h_{0}-T^{*} h_{0}+\left(A^{*}-i I\right)^{-1}(A+i I) \varphi \\
& =-2 i\left(A^{*}-i I\right)^{-1} h_{0}+\left(A^{*}-i I\right)^{-1}(A+i I) \varphi \\
& =\left(A^{*}-i I\right)^{-1}\left[(A+i I) \varphi-2 i h_{0}\right] .
\end{aligned}
$$

Therefore, $\psi \in \operatorname{Dom}\left(A^{*}\right)$ and

$$
(A+i I) \varphi-\left(A^{*}-i I\right) \psi=2 i h_{0}, \quad\left(h_{-}, h_{0}, h_{+}\right) \in \operatorname{Dom}\left(\mathscr{A}^{\prime}\right) .
$$

From (2.5) we obtain

$$
\begin{aligned}
T h_{+}(0) & =T\left(T^{*} h_{-}(0)+i \Gamma \varphi\right)=T T^{*} h_{-}(0)+i Q_{*} T(A+i I) \varphi \\
& =T T^{*} h_{-}(0)+i Q_{*}[(A+i I) \varphi-2 i \varphi] \\
& =T T^{*} h_{-}(0)+i \Gamma_{*} \psi+2 Q_{*}\left(\varphi-h_{0}\right) \\
& =T T^{*} h_{-}(0)+i \Gamma_{*} \psi+2 Q_{*}^{2} h_{-}(0)=h_{-}(0)+i \Gamma_{*} \psi,
\end{aligned}
$$

hence $\psi \in \operatorname{Dom}\left(A^{*}\right)$, so that $\operatorname{Dom}\left(\mathscr{A}^{\prime}\right) \subset \operatorname{Dom}\left(\mathscr{A}^{\prime \prime}\right)$. We leave the proof of inverse inclusion to the reader. The equality $\operatorname{Dom}\left(\mathscr{A}^{\prime}\right)=\operatorname{Dom}\left(\mathscr{A}^{\prime \prime}\right)$ and formula (2.5) now show that the mappings $\mathscr{A}^{\prime}$ and $\mathscr{A}^{\prime \prime}$ defined in (2.3) and (2.4) coincide on their domain. Therefore, $\mathscr{A}^{\prime}=\mathscr{A}^{\prime \prime}$.

In order to describe the resolvent of $\mathscr{A}$ let us introduce closed densely defined operators $\Upsilon_{ \pm}$and $\Upsilon_{ \pm}^{0}$ :

$$
\begin{array}{lll}
\Upsilon_{ \pm}:=i \frac{d}{d \xi}, & \operatorname{Dom}\left(\Upsilon_{+}\right):=W_{2}^{1}\left(\mathbb{R}_{+}, \mathcal{E}\right), & \operatorname{Dom}\left(\Upsilon_{-}\right):=W_{2}^{1}\left(\mathbb{R}_{-}, \mathcal{E}_{*}\right), \\
\Upsilon_{ \pm}^{0}:=i \frac{d}{d \xi}, & \operatorname{Dom}\left(\Upsilon_{+}^{0}\right):=\stackrel{\circ}{W_{2}^{1}}\left(\mathbb{R}_{+}, \mathcal{E}\right), & \operatorname{Dom}\left(\Upsilon_{-}^{0}\right):=\stackrel{\circ}{W}_{2}^{1}\left(\mathbb{R}_{-}, \mathcal{E}_{*}\right),
\end{array}
$$

where $W_{2}^{1}(\cdot, \cdot)$ and $\stackrel{\circ}{W} \underset{2}{1}(\cdot, \cdot)$ are the Sobolev classes. It is not difficult to show that $\left(\Upsilon_{ \pm}\right)^{*}=\Upsilon_{ \pm}^{0}$ and $\rho\left(\Upsilon_{+}\right)=\rho\left(\Upsilon_{-}^{0}\right)=\mathbb{C}_{+}, \rho\left(\Upsilon_{-}\right)=\rho\left(\Upsilon_{+}^{0}\right)=\mathbb{C}_{-}$. 
Theorem 2.4. For any $\left(h_{-}, h_{0}, h_{+}\right) \in \mathscr{H}$ the resolvent $(\mathscr{A}-z I)^{-1}, z \in \mathbb{C} \backslash \mathbb{R}$ is given by the formulae

(1) For $z \in \mathbb{C}_{-} \quad(\mathscr{A}-z I)^{-1}\left(h_{-}, h_{0}, h_{+}\right)$

$$
=\left(\begin{array}{c}
\psi_{-}(\xi) \\
(A-z I)^{-1}\left(h_{0}-\Gamma_{*}^{*} \psi_{-}(0)\right) \\
\left(\Upsilon_{+}^{0}-z I\right)^{-1} h_{+}+e^{-i z \xi}\left[\mathcal{S}^{*}(\bar{z}) \psi_{-}(0)+i \Gamma(A-z I)^{-1} h_{0}\right]
\end{array}\right)
$$

(2) For $z \in \mathbb{C}_{+} \quad(\mathscr{A}-z I)^{-1}\left(h_{-}, h_{0}, h_{+}\right)$

$$
=\left(\begin{array}{c}
\left(\Upsilon_{-}^{0}-z I\right)^{-1} h_{-}+e^{-i z \xi}\left[\mathcal{S}(z) \psi_{+}(0)-i \Gamma^{*}\left(A^{*}-z I\right)^{-1} h_{0}\right] \\
\left(A^{*}-z I\right)^{-1}\left(h_{0}-\Gamma^{*} \psi_{+}(0)\right) \\
\psi_{+}(\xi)
\end{array}\right)
$$

where $\psi_{ \pm}(\xi):=\left(\Upsilon_{ \pm}-z I\right)^{-1} h_{ \pm}, z \in \mathbb{C}_{ \pm}$. Symbols $(A-z I)^{-1} \Gamma_{*}^{*},\left(A^{*}-z I\right)^{-1} \Gamma^{*}$ here in in the sequel denote bounded in corresponding half planes $\mathbb{C}_{ \pm}$closures of operators $(A+i I)(A-z I)^{-1} Q_{*}$ and $\left(A^{*}-i I\right)\left(A^{*}-z I\right)^{-1} Q$ correspondingly. Bounded analytic vector functions $\mathcal{S}$ and $\mathcal{S}^{*}$ are are defined by the equalities

$$
\begin{aligned}
\mathcal{S}(z) & :=T-(z-i) \Gamma_{*}\left(A^{*}-z I\right)^{-1} Q, & & z \in \mathbb{C}_{+} \\
\mathcal{S}^{*}(\bar{z}) & :=T^{*}-(z+i) \Gamma(A-z I)^{-1} Q_{*}, & & z \in \mathbb{C}_{-}
\end{aligned}
$$

where $T=(A-i I)(A+i I)^{-1}$ is the Cayley transform of $A$ and $\mathcal{S}^{*}(\bar{z})=[\mathcal{S}(\bar{z})]^{*}$, $z \in \mathbb{C}_{-}$.

We omit the proof of Theorem 2.4. It consists in the direct verification of all the statements. It is convenient to use representation for $\mathscr{A}$ in the form (2.3) for $z \in \mathbb{C}_{-}$and in the form (2.4) for $z \in \mathbb{C}_{+}$. The interested reader is referred to [44] Theorem 2.2, where analogous calculations were carried out for a special case of operator $A$.

Statements of Theorem 2.4 show that apart from (2.2), the following identity for $A^{*}$ holds

$$
\left(A^{*}-z I\right)^{-1}=\left.P_{H}(\mathscr{A}-z I)^{-1}\right|_{H}, \quad z \in \mathbb{C}_{+},
$$

where $P_{H}=0 \oplus I_{H} \oplus 0$ is the orthogonal projection from $\mathscr{H}$ onto $H$.

Selfadjointness of $\mathscr{A}$ and equalities (2.2) and (2.6) result in the following important lemma.

Lemma 2.5 ([28, 32]). For any $u \in H$ functions $\Gamma(A-z I)^{-1} u$ and $\Gamma_{*}\left(A^{*}-z I\right)^{-1} u$ belong to the vector-valued Hardy classes $H_{2}^{-}(\mathcal{E})$ and $H_{2}^{+}\left(\mathcal{E}_{*}\right)$, correspondingly, with the norm estimates

$$
\left\|\Gamma(A-z I)^{-1} u\right\|_{H_{2}^{-}(\mathcal{E})} \leq \sqrt{2 \pi}\|u\|_{H}, \quad\left\|\Gamma_{*}\left(A^{*}-z I\right)^{-1} u\right\|_{H_{2}^{+}\left(\mathcal{E}_{*}\right)} \leq \sqrt{2 \pi}\|u\|_{H} .
$$

The proof is based on the relations for $\Psi_{A}[\cdot, \cdot]$ and $\Psi_{\left(-A^{*}\right)}[\cdot, \cdot]$ established in Lemma 2.1. Essentially, it repeats arguments of the work [28] (see [44] Lemma 2.4 as well) and is omitted here.

We conclude our review of results on the selfadjoint dilation of dissipative operator $A$ with the theorem equivalent to the completeness of "incomoing" and "outgoing" systems of eigenvectors of continuous spectrum of the dilation $\mathscr{A}$.

Consider two lineal manifolds in $\mathscr{H}$

$$
\mathscr{H}_{ \pm}:=\left\{(\mathscr{A}-z I)^{-1} \mathscr{D}_{ \pm} \mid z \in \mathbb{C}_{ \pm}\right\}
$$


Here and below we use the same notation for subspaces $\mathscr{D}_{ \pm}$and their imbeddings $\mathscr{D}_{-} \hookrightarrow \mathscr{D}_{-} \oplus 0 \oplus 0$ and $\mathscr{D}_{+} \hookrightarrow 0 \oplus 0 \oplus \mathscr{D}_{+}$into the dilation space $\mathscr{H}$.

Theorem 2.6. Let $H_{0} \subset H$ be the maximal space that reduces $A$ such that the restriction $\left.A\right|_{H_{0}}$ is selfadjoint. Then

$$
\operatorname{clos}\left(\mathscr{H}_{-} \vee \mathscr{H}_{+}\right)=\mathscr{H} \ominus H_{0}
$$

Proof. Denote $\mathscr{W}$ the set of finite linear combinations from $\mathscr{H}_{-} \vee \mathscr{H}_{+}$:

$$
\begin{aligned}
\mathscr{W}:= & \left\{\sum_{j=1}^{n} \alpha_{j}\left(\mathscr{A}-z_{j} I\right)^{-1} f_{j}+\sum_{s=1}^{m} \beta_{s}\left(\mathscr{A}-\zeta_{s} I\right)^{-1} g_{j}, \quad\right. \text { where } \\
\alpha_{j}, \beta_{s} \in \mathbb{C}, \quad z_{j} \in \mathbb{C}_{-}, \quad \zeta_{s} \in \mathbb{C}_{+}, & \\
& \left.f_{j} \in \mathscr{D}_{-}, \quad g_{s} \in \mathscr{D}_{+}, \quad j=1,2, \ldots n<\infty, \quad s=1,2, \ldots m<\infty\right\}
\end{aligned}
$$

We need to show that $\mathscr{W}$ is dense in $\mathscr{H} \ominus H_{0}$, or equivalently $\mathscr{W}^{\perp}=H_{0}$.

Since $s-\lim _{t \rightarrow+\infty}( \pm i t)(\mathscr{A} \pm i t I)^{-1}=I_{\mathscr{H}}$, we have $\mathscr{D}_{-} \oplus \mathscr{D}_{+} \subset \operatorname{clos}\left(\mathscr{H}_{-} \vee\right.$ $\left.\mathscr{H}_{+}\right)$. Therefore, $\mathscr{W}^{\perp} \subset H$. By the Hilbert identity, the set $\mathscr{W}$ is invariant for the resolvent $(\mathscr{A}-z I)^{-1}$, hence $\mathscr{W}^{\perp}$ is invariant for $\mathscr{A}$ as well due to selfadjointness of $\mathscr{A}$. From the dilation equalites $(2.3)$ and $(2.6)$ we obtain that $\left.(A-z I)^{-1}\right|_{\mathscr{W} \perp}$ coincides with the restriction $\left.(\mathscr{A}-z I)^{-1}\right|_{\mathscr{W} \perp}$ for $z \in \mathbb{C}_{ \pm}$. Hence, $\mathscr{W}^{\perp} \subset H_{0}$ by the definition of $H_{0}$.

Let us prove the inverse inclusion. For vector $w \in \mathscr{W}$ as in (2.7) denote $\psi_{j}:=$ $\left(\Upsilon_{-}-z_{j} I\right)^{-1} f_{j}$, and $\varphi_{s}:=\left(\Upsilon_{+}-\zeta_{s} I\right)^{-1} g_{s}$ where $j=1,2, \ldots n, s=1,2, \ldots m$. Then according to Theorem 2.4 for any $x \in H$ we have

$$
\begin{aligned}
&(w, x)=\left(\sum_{j} \alpha_{j}\left(\mathscr{A}-z_{j} I\right)^{-1} f_{j}+\sum_{s} \beta_{s}\left(\mathscr{A}-\zeta_{s} I\right)^{-1} g_{s}, x\right) \\
&=-\sum_{j} \alpha_{j}\left(\left(A-z_{j} I\right)^{-1} \Gamma_{*}^{*} \psi_{j}(0), x\right)-\sum_{s} \beta_{s}\left(\left(A^{*}-\zeta_{s} I\right)^{-1} \Gamma^{*} \varphi_{s}(0), x\right) \\
&=-\sum_{j} \alpha_{j}\left(Q_{*} \psi_{j}(0),\left(A^{*}-i I\right)\left(A^{*}-\bar{z}_{j} I\right)^{-1} x\right) \\
&-\sum_{s} \beta_{s}\left(Q \varphi_{s}(0),(A+i I)\left(A-\bar{\zeta}_{s} I\right)^{-1} x\right)
\end{aligned}
$$

If $x \in H_{0}$ then $x \perp \operatorname{Ran}(Q)$ and $x \perp \operatorname{Ran}\left(Q_{*}\right)$ by the definition of defect spaces, and $\left(A^{*}-i I\right)\left(A^{*}-\bar{z}_{j} I\right)^{-1} x \in H_{0},(A+i I)\left(A-\bar{\zeta}_{s} I\right)^{-1} x \in H_{0}$ since $H_{0}$ reduces $A$ and $A^{*}$. Hence $(w, x)=0$ and $H_{0} \subset \mathscr{W}^{\perp}$.

Corollary 2.7. In notation of Theorem 2.6 the set

$$
\begin{aligned}
\mathcal{W}:=\left\{\sum_{j=1}^{n} \alpha_{j}\left(A-z_{j} I\right)^{-1} \Gamma_{*}^{*} \psi_{j}+\sum_{s=1}^{m} \beta_{s}\left(A^{*}-\zeta_{s} I\right)^{-1} \Gamma^{*} \varphi_{s}, \quad\right. \text { where } \\
\alpha_{j}, \beta_{s} \in \mathbb{C}, \quad z_{j} \in \mathbb{C}_{-}, \quad \zeta_{s} \in \mathbb{C}_{+}, \\
\left.\psi_{j} \in \mathcal{E}_{*}, \quad \varphi_{s} \in \mathcal{E}, \quad j=1,2, \ldots n<\infty, \quad s=1,2, \ldots m<\infty\right\}
\end{aligned}
$$

is dense in $H \ominus H_{0}$. 
Proof. Sets $\left\{\varphi_{j}(0)\right\}$ and $\left\{\psi_{s}(0)\right\}$ in the Theorem proof coincide with $\mathcal{E}$ and $\mathcal{E}_{*}$ respectively by virtue of the Sobolev imbedding theorem. Hence, the set $\mathcal{W}$ is the projection of $\mathscr{W}$ onto $H$. The rest follows from the Theorem 2.6.

2.1.3. Characteristic function. A characteristic function of the Cayley transform of operator $A$, the contraction $T=(A-i I)(A+i I)^{-1}$, can be introduced by the formula (cf. [32]),

$$
\vartheta_{T}(z):=\left.\left(T-z \Delta_{T^{*}}\left(I-z T^{*}\right)^{-1} \Delta_{T}\right)\right|_{\mathcal{E}}, \quad z^{-1} \in \rho\left(T^{*}\right) .
$$

Function $\vartheta_{T}(z)$ is analytic in the unit disc $z \in \mathbb{D}$. Easily verifiable equalities

$$
\begin{aligned}
& I-\vartheta_{T}^{*}(\bar{\zeta}) \vartheta_{T}(z)=(1-z \zeta) \Delta_{T}(I-\zeta T)^{-1}\left(I-z T^{*}\right)^{-1} \Delta_{T} \\
& I-\vartheta_{T}(\zeta) \vartheta_{T}^{*}(\bar{z})=(1-z \zeta) \Delta_{T^{*}}\left(I-\zeta T^{*}\right)^{-1}(I-z T)^{-1} \Delta_{T^{*}}
\end{aligned}
$$

evaluated at $z=\bar{\zeta} \in \mathbb{D}$ show that values of $\vartheta_{T}$ are contractive operators that map $\mathcal{E}$ into $\mathcal{E}_{*}$. According to [47] we will call characteristic function of $A \in \mathscr{C}_{+}(H)$ the contractive in $\mathbb{C}_{+}$analytic operator function

$$
\mathcal{S}(z):=\vartheta_{T}\left(\frac{z-i}{z+i}\right), \quad T=(A-i I)(A+i I)^{-1} .
$$

Direct calculations based on (2.9) and (2.11) yield the representation (cf. Theorem 2.4)

$$
\mathcal{S}(z)=\left.\left(T+i(z-i) \Gamma_{*}\left(A^{*}-z I\right)^{-1} Q\right)\right|_{\mathcal{E}}, \quad z \in \rho\left(A^{*}\right) .
$$

It follows from the analyticity and contractiveness that there exist non-tangential boundary values of $\mathcal{S}$ in the strong operator topology almost everywhere on the real axis

$$
\mathcal{S}(k):=s-\lim _{\varepsilon \downarrow 0} \mathcal{S}(k-i \varepsilon), \quad \text { almost all } k \in \mathbb{R} .
$$

At that, $\|\mathcal{S}(k)\| \leq 1$ for almost all $k \in \mathbb{R}$ (see [32]). Moreover, it can be shown that the characteristic function $\mathcal{S}$ satisfies

$$
\mathcal{S}(z) \Gamma u=\Gamma_{*}\left(A^{*}-z I\right)^{-1}(A-z I) u, \quad z \in \rho\left(A^{*}\right), u \in \operatorname{Dom}(A) .
$$

and that the equation (2.12) determines the function $\mathcal{S}$ uniquely ([47]).

We conclude the characteristic function discussion with the formula for $\mathcal{S}^{*}$ obtained directly from its definition

$$
\mathcal{S}^{*}(\bar{z})=\left.\left(T^{*}-i(z+i) \Gamma(A-z I)^{-1} Q_{*}\right)\right|_{\mathcal{E}_{*}}, \quad z \in \rho(A) .
$$

Function $\mathcal{S}^{*}$ is contractive in the lower half-plane $\mathbb{C}_{-}$and almost everywhere on the real axis there exist its strong non-tangential boundary values $\mathcal{S}^{*}(k)$ that are contractive operators. Moreover, $\mathcal{S}^{*}(k)=\mathcal{S}(k)$ for almost all $k \in \mathbb{R}$ (see [32]).

2.2. Model construction. It is well known $([32,33,34])$ that for the model construction of operator $A$ it is sufficient to pass to a spectral representation of its dilation $\mathscr{A}$ so that $\mathscr{A}$ becomes the multiplication operator

$$
(\mathscr{A}-z I) \cong(k-z)^{-1}, \quad z \in \rho(\mathscr{A}), k \in \mathbb{R} .
$$

If such a representation is found, then according to (2.2), $A$ is unitarily equivalent to its functional model

$$
\begin{aligned}
(A-z I) & \left.\cong P_{H}(k-z)^{-1}\right|_{H}, & & z \in \mathbb{C}_{-}, k \in \mathbb{R} . \\
\left(A^{*}-z I\right) & \left.\cong P_{H}(k-z)^{-1}\right|_{H}, & & z \in \mathbb{C}_{+}, k \in \mathbb{R} .
\end{aligned}
$$


where $P_{H}$ is the projection to the image of $H$ under the spectral mapping. Here and in the sequel we use the same symbols for unitarily equivalent objects in hope that it would not lead to confusion. The construction of spectral mapping for $\mathscr{A}$ given below closely follows ideas of $[36,28,26]$. It is assumed everywhere that the operator $A$ is completely non-selfadjoint, that is, the subspace $H_{0}$ introduced in the Theorem 2.6 is trivial. Then the dilation $\mathscr{A}$ constructed in Theorem 2.3 is minimal, as follows from the Theorem 2.4 and density properties of exponents.

2.2.1. Model space. Following [36], [28] we arrive at the model Hilbert space $\mathbf{H}=$ $L_{2}\left(\begin{array}{cc}I & \mathcal{S}^{*} \\ \mathcal{S} & I\end{array}\right)$ by the factorization and subsequent completion of the linear manifold $\left\{\left(\begin{array}{l}\widetilde{g} \\ g\end{array}\right): \widetilde{g} \in L_{2}(\mathcal{E}), g \in L_{2}(\mathcal{E})\right\}$ of $\mathcal{E} \oplus \mathcal{E}_{*}$-valued vector functions with respect to the norm

$$
\left\|\left(\begin{array}{l}
\widetilde{g} \\
g
\end{array}\right)\right\|_{\mathbf{H}}^{2}:=\int_{\mathbb{R}}\left\langle\left(\begin{array}{cc}
I & \mathcal{S}^{*} \\
\mathcal{S} & I
\end{array}\right)\left(\begin{array}{l}
\widetilde{g} \\
g
\end{array}\right),\left(\begin{array}{l}
\widetilde{g} \\
g
\end{array}\right)\right\rangle_{\mathcal{E} \oplus \mathcal{E}_{*}} d k
$$

Note that, in general the completion operation makes it impossible to treat individual components $\widetilde{g}, g$ of a vector $\left(\begin{array}{c}\widetilde{g} \\ g\end{array}\right) \in \mathbf{H}$ as regular $L_{2}$-functions. However, two equivalent forms of the $\mathbf{H}$-norm

$$
\left\|\left(\begin{array}{c}
\widetilde{g} \\
g
\end{array}\right)\right\|_{\mathbf{H}}^{2}=\|\mathcal{S} \widetilde{g}+g\|_{L_{2}(\mathcal{E})}^{2}+\left\|\Delta_{*} g\right\|_{L_{2}(\mathcal{E})}^{2}=\left\|\widetilde{g}+\mathcal{S}^{*} g\right\|_{L_{2}(\mathcal{E})}^{2}+\|\Delta \widetilde{g}\|_{L_{2}(\mathcal{E})}^{2},
$$

where $\Delta:=\sqrt{I-\mathcal{S}^{*} \mathcal{S}}$ and $\Delta_{*}:=\sqrt{I-\mathcal{S S}^{*}}$ show that for each $\left(\begin{array}{l}\widetilde{g} \\ g\end{array}\right) \in \mathbf{H}$ expressions $\mathcal{S} \widetilde{g}+g, \widetilde{g}+\mathcal{S}^{*} g, \Delta \widetilde{g}$, and $\Delta_{*} g$ are in fact usual square summable vector-functions from $L_{2}(\mathcal{E})$ or $L_{2}\left(\mathcal{E}_{*}\right)$. Moreover, these equalities show that the right hand side of (2.14) is non-negative, therefore the norm $\|\cdot\|_{\mathbf{H}}$ is definite.

Operator-function $\left(\begin{array}{c}\widetilde{g} \\ g\end{array}\right) \mapsto(k-z)^{-1}\left(\begin{array}{c}\widetilde{g} \\ g\end{array}\right)$, where $\left(\begin{array}{l}\widetilde{g} \\ g\end{array}\right) \in \mathbf{H}, k \in \mathbb{R}$ is the independent variable, and $z \in \mathbb{C} \backslash \mathbb{R}$, is the resolvent of the multiplication operator $f \mapsto k f$, $f \in \mathbf{H}$ acting in $\mathbf{H}$. It is an absolutely continuous selfadjoint operator with the spectrum covering the whole real axis (cf. [32]).

Subspaces in $\mathbf{H}$

$$
\mathfrak{D}_{+}:=\left(\begin{array}{c}
H_{2}^{+}(\mathcal{E}) \\
0
\end{array}\right), \quad \mathfrak{D}_{-}:=\left(\begin{array}{c}
0 \\
H_{2}^{-}\left(\mathcal{E}_{*}\right)
\end{array}\right), \quad \mathfrak{H}:=\mathbf{H} \ominus\left[\mathfrak{D}_{+} \oplus \mathfrak{D}_{-}\right]
$$

where $H_{2}^{ \pm}\left(\mathcal{E}_{(*)}\right)$ are Hardy classes of $\mathcal{E}_{(*)}$-valued vector functions analytic in $\mathbb{C}_{ \pm}$, are mutually orthogonal. Here and in the following analytic functions from vectorvalued Hardy classes $H_{2}^{ \pm}(E)$ are equated with their boundary values existing almost everywhere on the real axis. These boundary values form two complementary orthogonal subspaces in $L_{2}(\mathbb{R}, E)=H_{2}^{+}(E) \oplus H_{2}^{-}(E)$. (See [41] for details.) The subspace $\mathfrak{H}$ can be described explicitly:

$$
\mathfrak{H}=\left\{\left(\begin{array}{c}
\widetilde{g} \\
g
\end{array}\right) \in \mathbf{H}: \widetilde{g}+\mathcal{S}^{*} g \in H_{2}^{-}(\mathcal{E}), \mathcal{S} \widetilde{g}+g \in H_{2}^{+}\left(\mathcal{E}_{*}\right)\right\}
$$

Orthogonal projection $P_{\mathfrak{H}}$ from $\mathbf{H}$ onto $\mathfrak{H}$ is defined by the following formula

$$
P_{\mathfrak{H}}\left(\begin{array}{c}
\widetilde{g} \\
g
\end{array}\right)=\left(\begin{array}{c}
\widetilde{g}-P_{+}\left(\widetilde{g}+\mathcal{S}^{*} g\right) \\
g-P_{-}(\mathcal{S} \widetilde{g}+g)
\end{array}\right), \quad \widetilde{g} \in L_{2}(\mathcal{E}), g \in L_{2}\left(\mathcal{E}_{*}\right)
$$

where $P_{ \pm}$are the orthogonal projections from $L_{2}$ onto Hardy classes $H_{2}^{ \pm}$. 
2.2.2. Spectral representation of dilation $\mathscr{A}$. In accordance with [28], introduce two linear mappings $\mathscr{F}_{+}: \mathscr{H} \rightarrow L_{2}(\mathbb{R}, \mathcal{E})$ and $\mathscr{F}_{-}: \mathscr{H} \rightarrow L_{2}\left(\mathbb{R}, \mathcal{E}_{*}\right)$

$$
\begin{aligned}
& \mathscr{F}_{+}: h \mapsto-\frac{1}{\sqrt{2 \pi}} \Gamma(A-k+i 0)^{-1} h_{0}+\mathcal{S}^{*}(k) \widehat{h}_{-}(k)+\widehat{h}_{+}(k) \\
& \mathscr{F}_{-}: h \mapsto-\frac{1}{\sqrt{2 \pi}} \Gamma_{*}\left(A^{*}-k-i 0\right)^{-1} h_{0}+\widehat{h}_{-}(k)+\mathcal{S}(k) \widehat{h}_{+}(k)
\end{aligned}
$$

where $h:=\left(h_{-}, h_{0}, h_{+}\right) \in \mathscr{H}$ and $\widehat{h}_{ \pm}$are the Fourier transforms of $h_{ \pm} \in \mathscr{D}_{ \pm}$extended by zero to the complementary semiaxis, $\widehat{h}_{ \pm}=(2 \pi)^{-1 / 2} \int_{\mathbb{R}_{ \pm}} h_{ \pm}(\xi) e^{i k \xi} d \xi$. By virtue of Paley-Wiener theorem, $\widehat{h}_{ \pm} \in H_{2}^{ \pm}$, see [41]. Due to Lemma 2.5 boundary values $\Gamma(A-k+i 0)^{-1} h_{0}$ and $\Gamma_{*}\left(A^{*}-k-i 0\right)^{-1} h_{0}$ exists for any $h_{0} \in H$ for almost all $k \in \mathbb{R}$ and belong to $L_{2}(\mathcal{E})$ and $L_{2}\left(\mathcal{E}_{*}\right)$ correspondingly. Moreover, according to this Lemma and boundedness of operator functions $\mathcal{S}(k), \mathcal{S}^{*}(k)$, mappings (2.16) are bounded as operators from $\mathscr{H}$ to $L_{2}(\mathcal{E})$ and $L_{2}\left(\mathcal{E}_{*}\right)$.

The distinguished role of $\mathscr{F}_{ \pm}$is revealed in the next Theorem where $\mathscr{A}$ is the minimal selfadjoint dilation of operator $A$.

Theorem 2.8 (Model Theorem). There exists an unique mapping $\Phi$ from the dilation space $\mathscr{H}$ onto the model space $\mathbf{H}$ with the properties:

(1) $\Phi$ is an isometry.

(2) $\widetilde{g}+\mathcal{S}^{*} g=\mathscr{F}_{+} h, \mathcal{S} \widetilde{g}+g=\mathscr{F}_{-} h$, where $\left(\begin{array}{l}\widetilde{g} \\ g\end{array}\right)=\Phi h, h \in \mathscr{H}$

(3) $\Phi \circ(\mathscr{A}-z I)^{-1}=(k-z)^{-1} \circ \Phi, \quad z \in \mathbb{C} \backslash \mathbb{R}$

(4) $\Phi H=\mathfrak{H}, \quad \Phi \mathscr{D}_{ \pm}=\mathfrak{D}_{ \pm}$

(5) $\mathscr{F}_{ \pm} \circ(\mathscr{A}-z I)^{-1}=(k-z I)^{-1} \circ \mathscr{F}_{ \pm}, \quad z \in \mathbb{C} \backslash \mathbb{R}$.

Property (3) means that $\Phi$ maps $\mathscr{A}$ into the multiplication operator on the space $\mathbf{H}$; therefore, the dissipative operator $A$ is mapped into its model representation (2.13), as required.

2.3. Notes on the Proof of Model Theorem. The proof of Theorem 2.8 is based on direct verification of all its statements for the spectral isometry $\Phi$ that maps $\mathscr{A}$ into the multiplication operator acting on $\mathbf{H}$. Following [28], we define $\Phi: \mathscr{H} \rightarrow \mathbf{H}$ initially on the set $\left(\mathscr{D}_{-}, \mathcal{W}, \mathscr{D}_{+}\right)$dense in $\mathscr{H}$. In notation of (2.8) let $\left(h_{-}, h_{0}, h_{+}\right) \in\left(\mathscr{D}_{-}, \mathcal{W}, \mathscr{D}_{+}\right)$, where

$$
h_{0}=\sum_{j=1}^{n} \alpha_{j}\left(A-z_{j} I\right)^{-1} \Gamma_{*}^{*} \psi_{j}+\sum_{s=1}^{m} \beta_{s}\left(A^{*}-\zeta_{s} I\right)^{-1} \Gamma^{*} \varphi_{s}
$$

We define the map $\Phi$ by

$$
\Phi:\left(\begin{array}{c}
h_{-} \\
h_{0} \\
h_{+}
\end{array}\right) \mapsto\left(\begin{array}{c}
\widehat{h}_{+}+\frac{i}{\sqrt{2 \pi}}\left[\sum_{j} \frac{\alpha_{j}}{k-z_{j}}\left[\mathcal{S}\left(\bar{z}_{j}\right)\right]^{*} \psi_{j}+\sum_{s} \frac{\beta_{s}}{k-\zeta_{s}} \varphi_{s}\right] \\
\widehat{h}_{-}-\frac{i}{\sqrt{2 \pi}}\left[\sum_{j} \frac{\alpha_{j}}{k-z_{j}} \psi_{j}+\sum_{s} \frac{\beta_{s}}{k-\zeta_{s}} \mathcal{S}\left(\zeta_{s}\right) \varphi_{s}\right]
\end{array}\right)
$$

Here $\widehat{h}_{ \pm}$are Fourier transforms of functions $h_{ \pm} \in L_{2}\left(\mathbb{R}_{ \pm}, E_{(*)}\right)$. Uniqueness of the map $\Phi$ that satisfies (2) follows directly from the definition of norm in $\mathbf{H}$. Secondly, equalities $\Phi \mathscr{D}_{ \pm}=\mathfrak{D}_{ \pm}$for $\Phi$ hold true by virtue of Paley-Wiener theorem. Moreover, since Fourier transforms $h_{ \pm} \mapsto \widehat{h}_{ \pm}$are isometric, restrictions $\left.\Phi\right|_{\mathscr{D}_{ \pm}}$are isometries onto $\mathfrak{D}_{ \pm}$. Property (3) follows from (2), (5), and the isometric property of of $\Phi$. The full proof of the Theorem goes beyond the scope of this paper. We refer the 
reader to works [28] and [44], where analogous calculations were carried out for special cases of dissipative operator $A$.

\section{NON-DISSIPATIVE OPERATOR}

3.1. Closed operators and $J$-spaces. Let $L \in \mathscr{C}(H)$ be a closed nonselfadjoint operator on the Hilbert space $H$ with the dense domain $\operatorname{Dom}(L)$ and non-empty resolvent set. Without any loss of generality we assume that $-i \in \rho(L)$. Then the Cayley transform of $L$

$$
V:=(L-i I)(L+i I)^{-1}=I-2 i(L+i I)^{-1}
$$

is the bounded operator on $H$. In what follows we assume that $L$ is not dissipative, or equivalently, $V$ is not a contraction, and that $L$ does not have nontrivial selfadjoint parts, which is equivalent to the triviality of the unitary part of $V$.

3.1.1. Characteristic function of operator $L$. The characteristic function of $V$ is analytic operator-valued function defined by

$$
\vartheta_{V}:=\left.\left(V-\zeta J_{*} \Delta_{V^{*}}\left(I-\zeta V^{*}\right)^{-1} \Delta_{V}\right)\right|_{E}, \quad \zeta^{-1} \in \rho\left(V^{*}\right)
$$

where operators

$$
\Delta_{V}:=\left|I-V^{*} V\right|^{1 / 2}, \quad \Delta_{V^{*}}:=\left|I-V V^{*}\right|^{1 / 2}, \quad J_{*}:=\operatorname{sign}\left(I-V V^{*}\right)
$$

are defined as functions of selfadjoint operators $I-V^{*} V$ and $I-V V^{*}$, cf. [7, 47]. Analogously to the case of dissipative operator $A$, the function $\vartheta_{V}$ acts between defect spaces $E:=\operatorname{clos} \operatorname{Ran}\left(\Delta_{V}\right)$ and $E_{*}:=\operatorname{clos} \operatorname{Ran}\left(\Delta_{V^{*}}\right), \vartheta_{V}: E \rightarrow E_{*}$. Introduce one more operator $J:=\operatorname{sign}\left(I-V^{*} V\right)$. Note that both $J$ and $J_{*}$ are involutions, that is, $J=J^{-1}=J^{*}, J_{*}=J_{*}^{-1}=J_{*}^{*}$ as mappings on $E$ and $E_{*}$. Moreover, $J \Delta_{V}=\Delta_{V} J$ and $J_{*} \Delta_{V^{*}}=\Delta_{V^{*}} J_{*}$. Direct calculations yield

$$
\begin{aligned}
& J-\left[\vartheta_{V}(z)\right]^{*} J_{*} \vartheta_{V}(\zeta)=(1-\bar{z} \zeta) \Delta_{V}(I-\bar{z} V)^{-1}\left(I-\zeta V^{*}\right)^{-1} \Delta_{V} \\
& J_{*}-\vartheta_{V}(z) J\left[\vartheta_{V}(\zeta)\right]^{*}=(1-z \bar{\zeta}) J_{*} \Delta_{V^{*}}\left(I-z V^{*}\right)^{-1}(I-\bar{\zeta} V)^{-1} J_{*} \Delta_{V^{*}}
\end{aligned}
$$

where $z^{-1}, \zeta^{-1} \in \rho\left(V^{*}\right)$. For $z=\zeta$ we have

$$
\begin{aligned}
& J-\left[\vartheta_{V}(\zeta)\right]^{*} J_{*} \vartheta_{V}(\zeta)=\left(1-|\zeta|^{2}\right) \Delta_{V}(I-\bar{\zeta} V)^{-1}\left(I-\zeta V^{*}\right)^{-1} \Delta_{V} \\
& J_{*}-\vartheta_{V}(\zeta) J\left[\vartheta_{V}(\zeta)\right]^{*}=\left(1-|\zeta|^{2}\right) J_{*} \Delta_{V^{*}}\left(I-\zeta V^{*}\right)^{-1}(I-\bar{\zeta} V)^{-1} J_{*} \Delta_{V_{*}}
\end{aligned}
$$

Similar to the definition of $\mathcal{S}(\cdot)$ via $\vartheta_{T}(\cdot)$ given in $(2.11)$, the characteristic function of $L$ is defined as the function $\Theta(z):=\vartheta_{V}\left((z-i)(z+i)^{-1}\right), z \in \rho\left(L^{*}\right)$. We will not require its expression in terms of $L$, even though it is not difficult to obtain by analogy with the case of dissipative operators explained earlier.

3.1.2. Potapov-Ginzburg transform. Introduce four orthogonal projections on the spaces $E$ and $E_{*}$ respectively, $X^{ \pm}:=\left(I_{E} \pm J\right) / 2$ and $X_{*}^{ \pm}:=\left(I_{E_{*}} \pm J_{*}\right) / 2$. Obviously, $I_{E}=X^{+}+X^{-}, J:=X^{+}-X^{-}$and the same relations are valid for $X_{*}^{ \pm}$and $E_{*}$. Involutions $J, J_{*}$ define indefinite products $J[\cdot, \cdot]$ and $J_{*}[\cdot, \cdot]$ on spaces $E, E_{*}$ by formulae

$$
\begin{gathered}
J[x, x]:=(J x, x)_{E}=\left\|X^{+} x\right\|^{2}-\left\|X^{-} x\right\|^{2}, \quad x \in E \\
J_{*}[y, y]:=\left(J_{*} y, y\right)_{E_{*}}=\left\|X_{*}^{+} y\right\|^{2}-\left\|X_{*}^{-} y\right\|^{2}, \quad y \in E_{*}
\end{gathered}
$$

where $(\cdot, \cdot)_{E},(\cdot, \cdot)_{E_{*}}$ are Hilbert scalar products in $E$ and $E_{*}$. With indefinite metrics induced by these products spaces $E$ and $E_{*}$ become so called Krein spaces 
(or $J$-spaces). For more detail on the theory of Krein spaces required in the sequel we refer the reader to [6] where further refrernces can be found.

Definition 3.1. Let $M: E \rightarrow E_{*}$ be a bounded operator. Suppose the equality $\operatorname{Ker}\left(\left.X_{*}^{-} M X^{-}\right|_{X^{-E}}\right)=\{0\}$ holds. The mapping $\omega(M)$ defined by the formula

$$
\omega(M):=\left(X^{-}+X_{*}^{+} M\right)\left(X^{+}+X_{*}^{-} M\right)^{-1}
$$

is called Potapov-Ginzburg transform (PG-transform) of $M$.

The PG-transform $\omega(M)$ is a bounded operator from $E_{+}:=X^{+} E \oplus X_{*}^{-} E_{*}$ to the space $E_{-}:=X^{-} E \oplus X_{*}^{+} E_{*}$. It is assumed that $E_{ \pm}$are endowed with Hilbert metrics, that is, $I_{E_{+}}:=X^{+}+X_{*}^{-}$and $I_{E_{-}}:=X^{-}+X_{*}^{+}$.

Definition 3.2. Operator $M: E \rightarrow E_{*}$ is called $\left(J J_{*}\right)$-bi-non-expansive (respectively, $\left(J J_{*}\right)$-bi-non-contractive) if for any $x \in E, y \in E_{*}$

$$
\begin{array}{r}
J_{*}[M x, M x] \leq J[x, x], \quad J\left[M^{*} y, M^{*} y\right] \leq J_{*}[y, y] \\
\left(J_{*}[M x, M x] \geq J[x, x], \quad J\left[M^{*} y, M^{*} y\right] \geq J_{*}[y, y]\right)
\end{array}
$$

Here $M^{*}$ is the adjoint of $M$ in the Hilbert metric of spaces $E, E_{*}$.

Let $M$ be $\left(J J_{*}\right)$-bi-non-expansive and $x \in X^{-} E$ is such that $X_{*}^{-} M x=0$. Then

$$
0 \leq\left\|X_{*}^{+} M x\right\|^{2}=J_{*}[M x, M x] \leq J[x, x]=-\left\|X^{-} x\right\|^{2} \leq 0 \Rightarrow x=0
$$

This simple observation shows that any $\left(J J_{*}\right)$-bi-non-expansive operator satisfies the condition of the PG-transform applicability from Definition 3.1 .

Theorem 3.3. The $P G$-transform $M \mapsto \omega(M)$ establishes a one-to-one correspondence between bounded $\left(J J_{*}\right)$-bi-non-expansive operators $M: E \rightarrow E_{*}$ and non-expansive operators (contractions) $W:=\omega(M): E_{+} \rightarrow E_{-}$satisfying condition $\operatorname{Ker}\left(\left.X^{-} W X_{*}^{-}\right|_{X_{*}^{-} E}\right)=\{0\}$. Moreover, $\operatorname{Ran}\left(X^{-} W X_{*}^{-}\right)=X^{-} E$.

Theorem 3.4. For any bounded $\left(J J_{*}\right)$-bi-non-expansive operator $M$ formulae

$$
\begin{aligned}
& \omega(M):=\left(X^{-}+X_{*}^{+} M\right)\left(X^{+}+X_{*}^{-} M\right)^{-1} \\
& \omega(M):=-\left(X_{*}^{+}-M X^{-}\right)^{-1}\left(X_{*}^{-}-M X^{+}\right)
\end{aligned}
$$

define the same operator. At that, the inversion formulae are valid

$$
\begin{aligned}
& M=\left(X_{*}^{-}+X_{*}^{+} W\right)\left(X^{+}+X^{-} W\right)^{-1} \\
& M=-\left(X_{*}^{+}-W X_{*}^{-}\right)^{-1}\left(X^{-}-W X^{+}\right)
\end{aligned}
$$

Proof of Theorem 3.3 can be found in [6], Chapter 5. Theorem 3.4 was proven in [16] for the case $E=E_{*}$. The general case is considered quite similarly by methods of linear relation theory [46]. Moreover, all factors in (3.3) and (3.4) are bounded operators and the formal passage to adjoint operators in these formulae yields correct results.

In notation of Theorem 3.4 following identities are verified directly

$$
\begin{gathered}
\left(X^{+}+X_{*}^{-} M\right)^{-1}=X^{+}+\left(X_{*}^{-} M X^{-}\right)^{-1}\left(X_{*}^{-}-X_{*}^{-} M X^{+}\right) \\
\left.\left(X^{-} W X_{*}^{-}\right)^{-1}\right|_{X^{-E}}=\left.X_{*}^{-} M X^{-}\right|_{X^{-E}}
\end{gathered}
$$

and

$$
\begin{aligned}
& \left(X^{+}+X_{*}^{-} M\right)^{-1}=X^{+}+X^{-} W \\
& \left(X_{*}^{+}-M X^{-}\right)^{-1}=X_{*}^{+}-W X_{*}^{-}
\end{aligned}
$$


Furthermore, for $W_{j}:=\omega\left(M_{j}\right), j=1,2$

$$
\begin{aligned}
I-W_{k}^{*} W_{j} & =\left(X^{+}+M_{k}^{*} X_{*}^{-}\right)^{-1}\left(J-M_{k}^{*} J_{*} M_{j}\right)\left(X^{+}+X_{*}^{-} M_{j}\right)^{-1} \\
I-W_{k} W_{j}^{*} & =\left(X_{*}^{+}-M_{k} X^{-}\right)^{-1}\left(J_{*}-M_{k} J M_{j}^{*}\right)\left(X_{*}^{+}-X^{-} M_{j}^{*}\right)^{-1} \\
J-M_{k}^{*} J_{*} M_{j} & =\left(X^{+}+W_{k}^{*} X^{-}\right)^{-1}\left(I-W_{k}^{*} W_{j}\right)\left(X^{+}+X^{-} W_{j}\right)^{-1} \\
J_{*}-M_{k} J M_{j}^{*} & =\left(X_{*}^{+}-W_{k} X_{*}^{-}\right)^{-1}\left(I-W_{k} W_{j}^{*}\right)\left(X_{*}^{+}-X_{*}^{-} W_{j}^{*}\right)^{-1}
\end{aligned}
$$

where $k, j=1,2$. Equations (3.6) illustrate the correspondence between metric properties of $\left(J J_{*}\right)$-bi-non-expansive operators $M_{1}, M_{2}$ and their PG-transforms, contractions $W_{j}=\omega\left(M_{j}\right), j=1,2$.

There exist a parallel version of PG-transform For $\left(J J_{*}\right)$-bi-non-contractive operators which formally can be obtained from statements of Theorem 3.3 and Theorem 3.4. In order to get correct results, one merely needs to exchange roles that the "negative" and "positive" projections play, $X^{-} \leftrightarrow X^{+}, X_{*}^{-} \leftrightarrow X_{*}^{+}$. Let us formulate a version of Theorem 3.4 for $\left(J J_{*}\right)$-bi-non-contractions.

Theorem 3.5. For any bounded $\left(J J_{*}\right)$-bi-non-contractive operator $M$ formulae

$$
\begin{aligned}
& \omega^{+}(M):=\left(X^{+}+X_{*}^{-} M\right)\left(X^{-}+X_{*}^{+} M\right)^{-1} \\
& \omega^{+}(M):=-\left(X_{*}^{-}-M X^{+}\right)^{-1}\left(X_{*}^{+}-M X^{-}\right)
\end{aligned}
$$

define the same operator. At that, the inversion formulae are valid

$$
\begin{aligned}
& M=\left(X_{*}^{+}+X_{*}^{-} W\right)\left(X^{-}+X^{+} W\right)^{-1} \\
& M=-\left(X_{*}^{-}-W X_{*}^{+}\right)^{-1}\left(X^{+}-W X^{-}\right)
\end{aligned}
$$

where $W=\omega^{+}(M)$.

Despite of expectations one may have, the PG-transform $\omega^{+}(M)$ defined in Theorem 3.5 is a contractive operator $\omega^{+}(M): E_{-} \rightarrow E_{+}$. This fact can be easily derived from definitions (3.7).

3.2. PG-transform of function $\vartheta_{V}$. Relations (3.2) show that values of the characteristic function $\vartheta_{V}(z)$ are $\left(J J_{*}\right)$-bi-non-expansive operators for $z^{-1} \in \rho\left(V^{*}\right)$, $|z|<1$. Therefore, for these $z \in \mathbb{D}$ the PG-transforms $\tau(z):=\omega\left(\vartheta_{V}(z)\right)$ is the analytic operator-function whose values are contractions from $E_{+}$to $E_{-}$.

The next Theorem plays the principal role in the model construction for operator $L$.

Theorem 3.6. In the notation introduced above:

(1) Contractive analytic operator function $\tau(z):=\omega\left(\vartheta_{V}(z)\right), z^{-1} \in \rho\left(V^{*}\right)$, $|z|<1$ can be written in the form

$$
\tau(z)=T_{11}+z T_{12}\left(I-z T_{22}\right)^{-1} T_{21},
$$

where $T_{11}: E_{+} \rightarrow E_{-}, T_{12}: H \rightarrow E_{-}, T_{21}: E_{+} \rightarrow H, T_{22}: H \rightarrow H$.

(2) Operator $T_{22}$ is a contraction, $\left\|T_{22}\right\| \leq 1$. Consequently, function $\tau(z)$ is analytic for $z \in \mathbb{D}$.

(3) Denote $T:=T_{22}^{*}$. Unitary parts of operators $V$ and $T$ coincide, and under assumptions of the paper, are trivial. 
(4) Let $\vartheta_{T}(z): \mathcal{E} \rightarrow \mathcal{E}_{*}, z \in \mathbb{D}$ be the characteristic function of the contractive operator $T:=T_{22}^{*}$ defined by (2.9). Then there exist two linear isometries $p: \mathcal{E} \rightarrow E_{+}$and $p^{\prime}: \mathcal{E}_{*} \rightarrow E_{-}$with the property

$$
\tau(z)=p^{\prime} \vartheta_{T}(z) p^{*}, \quad z^{-1} \in \rho\left(V^{*}\right),|z|<1 .
$$

Therefore, the operator function $\tau(z)$ is analytically extendable to the unit disc and its values are contractive operators from $E_{+}$to $E_{-}$for all $z \in \mathbb{D}$.

Proof. Proof of Theorem 3.6 is based on the results of D. Arov from [5] (see [6] for a concise account) and direct calulations.

(1) The claimed representation for $\tau(z)$ is valid for any bounded operatorfunction analytic in a neighborhood of the origin ([5]).

(2) Characteristic function $\vartheta_{V}(z)$ from (3.1) defines a bounded operator-matrix

$$
\mathbf{V}:=\left(\begin{array}{cc}
\left.V\right|_{E} & -J_{*} \Delta_{V^{*}} \\
\Delta_{V} & V^{*}
\end{array}\right): E \oplus H \rightarrow E_{*} \oplus H .
$$

Introduce involutions $\mathbf{J}, \mathbf{J}_{*}$ in Hilbert spaces $E \oplus H$ and $E_{*} \oplus H$ respectively by formulae $\mathbf{J}:=J \oplus I_{H}, \mathbf{J}_{*}:=J_{*} \oplus I_{H}$. These involutions induce the structure of Krein spaces in $E \oplus H$ and $E_{*} \oplus H$. Direct calculations show that operator $\mathbf{V}$ is $\left(\mathbf{J J}_{*}\right)$ unitary with respect to indefinite metrics generated by $\mathbf{J}$ and $\mathbf{J}_{*}$. In other words, $\mathbf{J}-\mathbf{V}^{*} \mathbf{J}_{*} \mathbf{V}=0$ and $\mathbf{J}_{*}-\mathbf{V} \mathbf{J} \mathbf{V}^{*}=0$. Since $\left(\mathbf{J J}_{*}\right)$-unitary operators are the special case of $\left(\mathbf{J J}_{*}\right)$-bi-non-expansive operators, Theorem 3.3 is applicable to $\mathbf{V}$ and $\mathbf{V}^{*}$. It follows from equalities (3.6) that the PG-transform of $\mathbf{V}$ denoted $\mathbf{T}:=\omega(\mathbf{V})$ is an isometry between $E_{+} \oplus H$ and $E_{-} \oplus H$. Calculations yield its precise form:

$$
\mathbf{T}=\left(\begin{array}{cc}
\omega\left(V_{0}\right) & -\left(X_{*}^{+}-V_{0} X^{-}\right)^{-1} J_{*} \Delta_{V^{*}} \\
\Delta_{V}\left(X^{+}+X_{*}^{-} V_{0}\right)^{-1} & V^{*}+\Delta_{V}\left(X_{*}^{-} V_{0} X^{-}\right)^{-1} X_{*}^{-} J_{*} \Delta_{V^{*}}
\end{array}\right)
$$

where we used notation $V_{0}:=\vartheta_{V}(0)=\left.V\right|_{E}$.

A fundamental result from [5] says that blocks of the PG-transform $\mathbf{T}$ of the operator $\mathbf{V}$ are exactly blocks in the representation for $\omega\left(\vartheta_{V}(z)\right)$ in the statement (1) of Theorem. In other words, operators $T_{11}, T_{12}, T_{21}$, and $T_{22}$ from statement (1) are given by (3.8):

$$
\begin{array}{ll}
T_{11}=\omega\left(V_{0}\right), & T_{12}=-\left(X_{*}^{+}-V_{0} X^{-}\right)^{-1} J_{*} \Delta_{V^{*}}, \\
T_{21}=\Delta_{V}\left(X^{+}+X_{*}^{-} V_{0}\right)^{-1}, & T_{22}=V^{*}+\Delta_{V}\left(X_{*}^{-} V_{0} X^{-}\right)^{-1} X_{*}^{-} J_{*} \Delta_{V^{*}}
\end{array}
$$

According to properties of PG-transform, operators $\mathbf{T}$ and $\mathbf{T}^{*}$ are isometries in Hilbert norms, $\mathbf{T}^{*} \mathbf{T}=I_{E_{+} \oplus H}, \mathbf{T} \mathbf{T}^{*}=I_{E_{-} \oplus H}$. Detailed form of these identities is

$$
\begin{aligned}
& \left(\begin{array}{cc}
I_{E_{+}} & 0 \\
0 & I_{H}
\end{array}\right)=\left(\begin{array}{cc}
T_{11}^{*} T_{11}+T_{21}^{*} T_{21} & T_{11}^{*} T_{12}+T_{21}^{*} T_{22} \\
T_{12}^{*} T_{11}+T_{22}^{*} T_{21} & T_{12}^{*} T_{12}+T_{22}^{*} T_{22}
\end{array}\right) \\
& \left(\begin{array}{cc}
I_{E_{-}} & 0 \\
0 & I_{H}
\end{array}\right)=\left(\begin{array}{ll}
T_{11} T_{11}^{*}+T_{12} T_{12}^{*} & T_{11} T_{21}^{*}+T_{12} T_{22}^{*} \\
T_{21} T_{11}^{*}+T_{22} T_{12}^{*} & T_{21} T_{21}^{*}+T_{22} T_{22}^{*}
\end{array}\right)
\end{aligned}
$$

An immediate consequence of (3.10)

$$
I-T_{22}^{*} T_{22}=T_{12}^{*} T_{12} \geq 0, \quad I-T_{22} T_{22}^{*}=T_{21} T_{21}^{*} \geq 0
$$

shows that both operator $T_{22}$ and its adjoint $T_{22}^{*}$ are contractions. 
(3) Denote $T:=T_{22}^{*}$. It is a contractive operator on $H$. Due to (3.11) and (3.9) the defect operators (2.1) of $T$ are

$$
\begin{aligned}
\Delta_{T}^{2} & =I-T_{22} T_{22}^{*}=\Delta_{V}\left(X^{+}+X_{*}^{-} V_{0}\right)^{-1}\left(X^{+}+V_{0}^{*} X_{*}^{-}\right)^{-1} \Delta_{V} \\
\Delta_{T^{*}}^{2} & =I-T_{22}^{*} T_{22}=J_{*} \Delta_{V^{*}}\left(X_{*}^{+}-X^{-} V_{0}^{*}\right)^{-1}\left(X_{*}^{+}-V_{0} X^{-}\right)^{-1} J_{*} \Delta_{V^{*}}
\end{aligned}
$$

Therefore, there exist two isometries $p: \mathcal{E} \rightarrow E$ and $p^{\prime}: \mathcal{E}_{*} \rightarrow E_{*}$, where $\mathcal{E}, \mathcal{E}_{*}$ are defect subspaces of $T$, such that

$$
\begin{aligned}
& p \Delta_{T}=T_{21}^{*}=\left(X^{+}+V_{0}^{*} X_{*}^{-}\right)^{-1} \Delta_{V}, \\
& p^{\prime} \Delta_{T^{*}}=-T_{12}=\left(X_{*}^{+}-V_{0} X^{-}\right)^{-1} J_{*} \Delta_{V^{*}}
\end{aligned}
$$

Further, due to (3.9) operator $T$ has the form

$$
T:=V+J_{*} \Delta_{V^{*}}\left(X^{-} V_{0}^{*} X_{*}^{-}\right)^{-1} X^{-} \Delta_{V}
$$

Now, if $H_{0} \subset H$ is the space that reduces $V$ and the part $\left.V\right|_{H_{0}}$ is unitary, then $\left.\Delta_{V}\right|_{H_{0}}=0$ and $\left.\Delta_{V^{*}}\right|_{H_{0}}=0$. It follows from (3.12) that $\left.\Delta_{T}\right|_{H_{0}}=\left.\Delta_{T^{*}}\right|_{H_{0}}=$ 0 . Moreover, according to (3.13) operator $\left.T\right|_{H_{0}}$ coincides with $\left.V\right|_{H_{0}}$, therefore is unitary. Obviously, operators $T$ and $V$ in this argument can be swapped, and that completes the proof of statement (3).

(4) Characteristic function $\vartheta_{T}(z),|z|<1$ of the contraction $T$ is defined in (2.9). Its values are contractive operators acting between defect subspaces $\mathcal{E}, \mathcal{E}_{*}$ of $T$. Passing to adjoint operators in (3.12) and taking into consideration invertibility of terms in (3.12) we have $\operatorname{Ran}\left(\Delta_{T}\right)=\operatorname{Ran}\left(\Delta_{V}\right), \operatorname{Ran}\left(\Delta_{T^{*}}\right)=\operatorname{Ran}\left(\Delta_{V^{*}}\right)$. Therefore, the defect subspaces of $T$ and $V$ coincide: $E=\mathcal{E}, E_{*}=\mathcal{E}_{*}$. Next, for $|z|<1$ we obtain in accordance with (3.12),

$$
\begin{aligned}
p^{\prime} \vartheta_{T}(z) p^{*} & =p^{\prime}\left(\left.T\right|_{\mathcal{E}}\right) p^{*}-z p^{\prime} \Delta_{T^{*}}\left(I-z T^{*}\right)^{-1} \Delta_{T} p^{*} \\
& =p^{\prime}\left(\left.T\right|_{E}\right) p^{*}+z T_{12}\left(I-z T_{22}\right)^{-1} T_{21}
\end{aligned}
$$

and we only need to show that $p^{\prime}\left(\left.T\right|_{E}\right) p^{*}=T_{11}$. Let use one of the identities (3.10) again. Due to (3.12) and interwining property $T \Delta_{T}=\Delta_{T^{*}} T$,

$$
0=T_{11} T_{21}^{*}+T_{12} T_{22}^{*}=T_{11} p \Delta_{T}-p^{\prime} \Delta_{T^{*}} T=\left(T_{11} p-p^{\prime} T\right) \Delta_{T} .
$$

Therefore, $T_{11} p x=p^{\prime} T x$ for any $x \in \operatorname{Ran}\left(\Delta_{T}\right)$. Since the range $\operatorname{Ran}\left(\Delta_{T}\right)$ is dense in $E=\mathcal{E}$, all operators here are bounded, and $p$ is an isometry, we finally obtain $T_{11}=p^{\prime}\left(\left.T\right|_{E}\right) p^{*}$.

The proof is complete.

Analogous theory can be developed for values of characteristic function $\vartheta_{V}(z)$, $z^{-1} \in \rho\left(V^{*}\right)$ outside of the unit disc, $|z|>1$. As equalities (3.2) show, operators $\vartheta_{V}(z)$ in this case are $\left(J J_{*}\right)$-bi-non-contractive according to Definition 3.2. Therefore, Theorem 3.5 is applicable and analytic operator-function $\omega^{+}\left(\vartheta_{V}(z)\right)$, $z^{-1} \in \rho\left(V^{*}\right),|z|>1$ is contractive. Next result establishes a close relationship between this function and $\tau(\cdot)$ defined in Theorem 3.6.

Theorem 3.7. For any $z \in \rho(V) \cap \mathbb{D}$

$$
\begin{aligned}
\vartheta_{V}(1 / \bar{z}) & =\left(X_{*}^{+}+X_{*}^{-} \tau^{*}(z)\right)\left(X^{-}+X^{+} \tau^{*}(z)\right)^{-1} \\
\vartheta_{V}(1 / \bar{z}) & =-\left(X_{*}^{-}-\tau^{*}(z) X_{*}^{+}\right)^{-1}\left(X^{+}-\tau^{*}(z) X^{-}\right) \\
\tau^{*}(z) & =\left(X^{+}+X_{*}^{-} \vartheta_{V}(1 / \bar{z})\right)\left(X^{-}+X_{*}^{+} \vartheta_{V}(1 / \bar{z})\right)^{-1} \\
\tau^{*}(z) & =-\left(X_{*}^{-}-\vartheta_{V}(1 / \bar{z}) X^{+}\right)^{-1}\left(X_{*}^{+}-\vartheta_{V}(1 / \bar{z}) X^{-}\right)
\end{aligned}
$$


or equivalently,

$$
\omega^{+}\left(\vartheta_{V}(1 / \bar{z})\right)=\tau^{*}(z), \quad z \in \rho(V) \cap \mathbb{D} .
$$

It is convenient to postpone the proof of Theorem 3.7 until more results on resolvents of $V$ and $T$ become available. At this moment we only note that equalities (3.14) are equivalent to one another due to properties of transform $\omega^{+}$ explained in Theorem 3.5. Furthermore, any of them yield the claimed identity $\omega^{+}\left(\vartheta_{V}(1 / \bar{z})\right)=\tau^{*}(z)$ due to uniqueness of the PG-transform.

3.3. Resolvent identities. Resolvents of operators $V$ and $T$ are related via usual Hilbert identities. We need their variant

$$
(I-z T)^{-1}-(I-z V)^{-1}=z(I-z V)^{-1}(T-V)(I-z T)^{-1}
$$

where $z \in \mathbb{D}$ in some neighborhood of the origin. According to (3.12) the difference $T-V=J_{*} \Delta_{V^{*}}\left(X^{-} V_{0}^{*} X_{*}^{-}\right)^{-1} X^{-} \Delta_{V}$ from (3.13) can be recast into the form

$$
T-V=J_{*} \Delta_{V^{*}}\left(X^{-} V_{0}^{*} X_{*}^{-}\right)^{-1} X^{-}\left(X^{+}+V_{0}^{*} X_{*}^{-}\right) p \Delta_{T}=J_{*} \Delta_{V^{*}} X_{*}^{-} p \Delta_{T}
$$

Therefore, from (3.15) we obtain

$$
p \Delta_{T}(I-z V)^{-1}=\left[I-z p \Delta_{T}(I-z V)^{-1} J_{*} \Delta_{V^{*}} X_{*}^{-}\right] p \Delta_{T}(I-z T)^{-1}
$$

and with help of (3.12),

$$
\begin{aligned}
& \Delta_{V}(I-z V)^{-1}=\left(X^{+}+V_{0}^{*} X_{*}^{-}\right) p \Delta_{T}(I-z V)^{-1} \\
& =\left[X^{+}+V_{0}^{*} X_{*}^{-}-z \Delta_{V}(I-z V)^{-1} J_{*} \Delta_{V^{*}} X_{*}^{-}\right] p \Delta_{T}(I-z T)^{-1} \\
& =\left[X^{+}+\left[\vartheta_{V}(\bar{z})\right]^{*} X_{*}^{-}\right] p \Delta_{T}(I-z T)^{-1} .
\end{aligned}
$$

Quite analogously, since

$$
\left(I-z T^{*}\right)^{-1}-\left(I-z V^{*}\right)^{-1}=z\left(I-z V^{*}\right)^{-1}\left(T^{*}-V^{*}\right)\left(I-z T^{*}\right)^{-1}
$$

and

$$
T^{*}-V^{*}=\Delta_{V}\left(X_{*}^{-} V_{0} X^{-}\right)^{-1} X_{*}^{-} J_{*} \Delta_{V^{*}}=-\Delta_{V} X^{-} p^{\prime} \Delta_{T^{*}}
$$

we have

$$
p^{\prime} \Delta_{T^{*}}\left(I-z V^{*}\right)^{-1}=\left[I+z p^{\prime} \Delta_{T^{*}}\left(I-z V^{*}\right)^{-1} \Delta_{V} X^{-}\right] p^{\prime} \Delta_{T^{*}}\left(I-z T^{*}\right)^{-1} .
$$

Therefore,

$$
\begin{aligned}
& J_{*} \Delta_{V^{*}}\left(I-z V^{*}\right)^{-1}=\left(X_{*}^{+}-V_{0} X^{-}\right) p^{\prime} \Delta_{T^{*}}\left(I-z V^{*}\right)^{-1} \\
& =\left[X_{*}^{+}-V_{0} X^{-}+z J_{*} \Delta_{V^{*}}\left(I-z V^{*}\right)^{-1} \Delta_{V} X^{-}\right] p^{\prime} \Delta_{T^{*}}\left(I-z T^{*}\right)^{-1} \\
& =\left[X_{*}^{+}-\vartheta_{V}(z) X^{-}\right] p^{\prime} \Delta_{T^{*}}\left(I-z T^{*}\right)^{-1} .
\end{aligned}
$$

Formulae (3.16) and (3.17) comprise first two statements of the next Theorem

Theorem 3.8. For any $z \in \mathbb{D}$ the "resolvent identities" hold

$$
\begin{aligned}
p \Delta_{T}(I-z T)^{-1} & =\left[X^{+}+\vartheta_{V}^{*}(\bar{z}) X_{*}^{-}\right]^{-1} \Delta_{V}(I-z V)^{-1} \\
p^{\prime} \Delta_{T^{*}}\left(I-z T^{*}\right)^{-1} & =\left[X_{*}^{+}-\vartheta_{V}(z) X^{-}\right]^{-1} J_{*} \Delta_{V^{*}}\left(I-z V^{*}\right)^{-1} \\
p^{\prime} \Delta_{T^{*}}\left(I-z T^{*}\right)^{-1}(V-z I) & =\left[X^{-}+\tau(z) X^{+}\right] \Delta_{V} \\
p \Delta_{T}(I-z T)^{-1}\left(V^{*}-z I\right) & =\left[-X_{*}^{-}+\tau^{*}(\bar{z}) X_{*}^{+}\right] J_{*} \Delta_{V^{*}}
\end{aligned}
$$


Proof. The right hand side of first two equalities are analytic functions for all $z \in \mathbb{D}$ since the left hand sides are analytic in the whole of unit disc. Their correctness in a neighborhood of the origin is proven by (3.16) and (3.17). The rest follows from the analyticity.

In order to verify two last statements we need the following relation for the characteristic function $\vartheta_{V}(\cdot)$,

$$
\vartheta_{V}(z) J \Delta_{V}=J_{*} \Delta_{V^{*}}\left(I-z V^{*}\right)^{-1}(V-z I), \quad z^{-1} \in \rho\left(V^{*}\right),|z|<1
$$

Its proof is a straightforward exercise and we omit it here. Let us now consider the third formula in the Theorem's statement. We have

$$
\begin{aligned}
p^{\prime} \Delta_{T^{*}}(I & \left.-z T^{*}\right)^{-1}(V-z I) \\
& =\left(X_{*}^{+}-\vartheta_{V}(z) X^{-}\right)^{-1} J_{*} \Delta_{V^{*}}\left(I-z V^{*}\right)^{-1}(V-z I) \\
& =\left(X_{*}^{+}-\vartheta_{V}(z) X^{-}\right)^{-1} \vartheta_{V}(z) J \Delta_{V}
\end{aligned}
$$

From the other side, according to the definition of PG-transform (3.3)

$$
\begin{aligned}
\left(X^{-}\right. & \left.+\tau(z) X^{+}\right) \Delta_{V} \\
& =\left[X^{-}-\left(X_{*}^{+}-\vartheta_{V}(z) X^{-}\right)^{-1}\left(X_{*}^{-}-\vartheta_{V}(z) X^{+}\right) X^{+}\right] \Delta_{V} \\
& =\left(X_{*}^{+}-\vartheta_{V}(z) X^{-}\right)^{-1}\left[-\vartheta_{V}(z) X^{-}+\vartheta_{V}(z) X^{+}\right] \Delta_{V} \\
& =\left(X_{*}^{+}-\vartheta_{V}(z) X^{-}\right)^{-1} \vartheta_{V}(z) J \Delta_{V},
\end{aligned}
$$

as required. The last equality in the Theorem claim is verified analogously.

The proof is complete.

Now we are in position to prove Theorem 3.7.

Proof of Theorem 3.7. Let us check the first equality (3.14) rewritten in the form

$$
\vartheta_{V}(1 / \bar{z})\left(X^{-}+X^{+} \tau^{*}(z)\right)=X_{*}^{+}+X_{*}^{-} \tau^{*}(z), \quad z \in \rho(V) \cap \mathbb{D} .
$$

We start with the expression for $\vartheta_{V}(1 / \bar{z})$ resulting from (3.12)

$$
\begin{aligned}
\vartheta_{V}(1 / \bar{z}) & =V_{0}-(1 / \bar{z}) J_{*} \Delta_{V^{*}}\left(I-(1 / \bar{z}) V^{*}\right)^{-1} \Delta_{V} \\
& =V_{0}+J_{*} \Delta_{V^{*}}\left(V^{*}-\bar{z} I\right)^{-1} \Delta_{V} \\
& =V_{0}+\left(X_{*}^{+}-V_{0} X^{-}\right) p^{\prime} \Delta_{T^{*}}\left(V^{*}-\bar{z} I\right)^{-1} \Delta_{V}
\end{aligned}
$$

Next, by passing to adjoint operators in the third formula of Theorem 3.8 we obtain

$$
\Delta_{V}\left(X^{-}+X^{+} \tau^{*}(z)\right)=\left(V^{*}-\bar{z} I\right)(I-\bar{z} T)^{-1} \Delta_{T^{*}}\left(p^{\prime}\right)^{*}
$$

The last ingredient of the proof is the second identity (2.10) for $\zeta=0$

$$
I-T_{0} \vartheta^{*}(\bar{z})=\Delta_{T^{*}}(I-z T)^{-1} \Delta_{T^{*}}
$$

and its consequence due to statement (4) of Theorem 3.6

$$
I-\tau(0) \tau^{*}(z)=I-\omega\left(V_{0}\right) \tau^{*}(z)=p^{\prime} \Delta_{T^{*}}(I-\bar{z} T)^{-1} \Delta_{T^{*}}\left(p^{\prime}\right)^{*} .
$$


where $V_{0}:=\left.V\right|_{E}$. Now we have for the left hand side of (3.18)

$$
\begin{aligned}
\vartheta_{V} & (1 / \bar{z})\left(X^{-}+X^{+} \tau^{*}(z)\right) \\
& =\left[V_{0}+\left(X_{*}^{+}-V_{0} X^{-}\right) p^{\prime} \Delta_{T^{*}}\left(V^{*}-\bar{z} I\right)^{-1} \Delta_{V}\right]\left(X^{-}+X^{+} \tau^{*}(z)\right) \\
& =V_{0}\left(X^{-}+X^{+} \tau^{*}(z)\right)+\left(X_{*}^{+}-V_{0} X^{-}\right) p^{\prime} \Delta_{T^{*}}(I-\bar{z} T)^{-1} \Delta_{T^{*}}\left(p^{\prime}\right)^{*} \\
& =V_{0}\left(X^{-}+X^{+} \tau^{*}(z)\right)+\left(X_{*}^{+}-V_{0} X^{-}\right)\left(I-\tau(0) \tau^{*}(z)\right) \\
& =X_{*}^{+}+\left[V_{0} X^{+}-\left(X_{*}^{+}-V_{0} X^{-}\right) \tau(0)\right] \tau^{*}(z) \\
& =X_{*}^{+}+\left[V_{0} X^{+}+\left(X_{*}^{-}-V_{0} X^{+}\right)\right] \tau^{*}(z)=X_{*}^{+}+X_{*}^{-} \tau^{*}(z),
\end{aligned}
$$

where on the last step we used the second definition (3.3) for $\tau(0)=\omega\left(V_{0}\right)$. Equality (3.18) is obtained, which completes the proof.

Corollary 3.9. Theorem 3.7 shows that operator functions on the right hand side of two last resolvent identities of Theorem 3.8 are boundedly invertible for suitable values of $z \in \mathbb{D}$. More precisely,

$$
\begin{array}{ll}
p^{\prime} \Delta_{T^{*}}\left(I-z T^{*}\right)^{-1}=\left[X^{-}+\vartheta_{V}^{*}(1 / \bar{z}) X_{*}^{+}\right]^{-1} \Delta_{V}(V-z I)^{-1}, & z \in \rho(V) \cap \mathbb{D} \\
p \Delta_{T}(I-z T)^{-1}=\left[-X_{*}^{-}+\vartheta_{V}(1 / z)\right]^{-1} J_{*} \Delta_{V^{*}}\left(V^{*}-z I\right)^{-1}, & z \in \rho\left(V^{*}\right) \cap \mathbb{D}
\end{array}
$$

where

$$
\begin{gathered}
\left(X^{-}+\vartheta_{V}^{*}(1 / \bar{z}) X_{*}^{+}\right)^{-1}=X^{-}+\tau(z) X^{+}, \quad z \in \rho(V) \cap \mathbb{D} \\
\left(-X_{*}^{-}+\vartheta_{V}(1 / z) X^{+}\right)^{-1}=-X_{*}^{-}+\tau^{*}(\bar{z}) X_{*}^{+}, \quad z \in \rho\left(V^{*}\right) \cap \mathbb{D}
\end{gathered}
$$

according to analogs of properties (3.6) for PG-transform $\omega^{+}$.

3.4. Dissipative operator and factorizations of characteristic function. Now we return to the initial setting of non-bounded operators. Recall that $L$ is completely non-selfadjoint non-dissipative operator with the characteristic operator function $\Theta(z):=\vartheta_{V}\left((z-i)(z+i)^{-1}\right)$ analytic in some neighborhood of $z=i$. Let us show that the operator $T$ introduced in (3.13) is a Cayley transform of some completely non-selfadjoint operator $A \in \mathscr{C}^{+}(H)$. To that end we only have to show that $(T-I)$ is invertible. Then $A:=(-i)(T+I)(T-I)^{-1}$ defined on $\operatorname{Ran}(A):=\operatorname{Ran}(T-I)$ satisfies all conditions listed above. Theorem 3.6 ensures that $T$ is completely non-unitary. By duality, so is the adjoint $T^{*}$. Therefore, $z=1$ does not belong to the point spectrum of $T$ and $T^{*}$. Otherwise, the part of $T$ in corresponding eigenspace would be an unitary. Since $\operatorname{Ker}\left(T^{*}-I\right)=\{0\}$, the range $\operatorname{Ran}(T-I)$ is dense in $H$. On the other hand, $\operatorname{Ker}(T-I)=\{0\}$ means that there exists an unbounded inverse $(T-I)^{-1}$ defined on the dense set $\operatorname{Ran}(T-I)$. This inverse is a closed operator because $T$ is defined everywhere in $H$.

Let $\mathcal{S}(z), z \in \mathbb{C}_{+}$be the characteristic function of $A:=(-i)(T+I)(T-I)^{-1}$. Due to the definition (2.11) and Theorem 3.6, $\tau((z-i) /(z+i))=p^{\prime} \mathcal{S}(z) p^{*}$ for $z \in \mathbb{C}_{+}$. Introduce bounded analytic function

$$
S(z)=\tau((z-i) /(z+i))=p^{\prime} \mathcal{S}(z) p^{*}, \quad z \in \mathbb{C}_{+}
$$

According to [47], $S(\cdot)$ is called characteristic function of $A$ as well. Obviously, function $S(\cdot)$ can be used to construct the Sz.-Nagy-Foiaş model for $A$ in the same manner as the function $\mathcal{S}(\cdot)$ was used in Section 2 . The only difference is the choice of defect spaces. In place of $\mathcal{E}, \mathcal{E}_{*}$ one should use $E_{+}$and $E_{-}$, respectively. 
Let us rewrite relationships between characteristic functions $\Theta$ and $S$ stated in Theorems 3.4, 3.5, and 3.7. For $\zeta \in \rho\left(L^{*}\right) \cap \mathbb{C}_{-}$

$$
\begin{aligned}
\Theta(\zeta) & =\left(X_{*}^{+}+X_{*}^{-} S^{*}(\bar{\zeta})\right)\left(X^{-}+X^{+} S^{*}(\bar{\zeta})\right)^{-1} \\
\Theta(\zeta) & =-\left(X_{*}^{-}-S^{*}(\bar{\zeta}) X_{*}^{+}\right)\left(X^{+}-S^{*}(\bar{\zeta}) X^{-}\right)^{-1} \\
S^{*}(\bar{\zeta}) & =\left(X^{+}+X_{*}^{-} \Theta(\zeta)\right)\left(X^{-}+X_{*}^{+} \Theta(\zeta)\right)^{-1} \\
S^{*}(\bar{\zeta}) & =-\left(X_{*}^{-}-\Theta(\zeta) X^{+}\right)^{-1}\left(X_{*}^{+}-\Theta(\zeta) X^{-}\right)
\end{aligned}
$$

and for $z \in \rho\left(L^{*}\right) \cap \mathbb{C}_{+}$

$$
\begin{aligned}
& \Theta(z)=\left(X_{*}^{-}+X_{*}^{+} S(z)\right)\left(X^{+}+X^{-} S(z)\right)^{-1} \\
& \Theta(z)=-\left(X_{*}^{+}-S(z) X_{*}^{-}\right)\left(X^{-}-S(z) X^{+}\right)^{-1} \\
& S(z)=\left(X^{-}+X_{*}^{+} \Theta(z)\right)\left(X^{+}+X_{*}^{-} \Theta(z)\right)^{-1} \\
& S(z)=-\left(X_{*}^{+}-\Theta(z) X^{-}\right)^{-1}\left(X_{*}^{-}-\Theta(z) X^{+}\right)
\end{aligned}
$$

These relations represent characteristic functions $\Theta, S$ in form of factorizations by two bounded analytic operator functions in corresponding domains of the complex plane.

The next set of equalities follows from properties of PG-transforms $\omega$ and $\omega^{+}$ (cf. Corollary 3.9). For $z \in \rho\left(L^{*}\right) \cap \mathbb{C}_{+}$and $\zeta \in \rho\left(L^{*}\right) \cap \mathbb{C}_{-}$,

$$
\begin{aligned}
\left(X^{+}+X_{*}^{-} \Theta(z)\right)^{-1} & =X^{+}+X^{-} S(z), \\
\left(X_{*}^{+}-\Theta(z) X^{-}\right)^{-1} & =X_{*}^{+}-S(z) X_{*}^{-}, \\
\left(X^{-}+X_{*}^{+} \Theta(\zeta)\right)^{-1} & =X^{-}+X^{+} S^{*}(\bar{\zeta}), \\
\left(X_{*}^{-}-\Theta(\zeta) X^{+}\right)^{-1} & =X_{*}^{-}-S^{*}(\bar{\zeta}) X_{*}^{+}
\end{aligned}
$$

Remark that relations obtained from (3.19), (3.20), and (3.21) by the formal passage to adjoint operators hold true. We will use the special notation for the bounded analytic operator functions defined on the right hand side of (3.21)

$$
\begin{array}{lll}
\Theta_{1}(z):=X^{-}+S(z) X^{+}: E \rightarrow E_{-}, & z \in \mathbb{C}_{+} \\
\Theta_{2}(z):=-X_{*}^{-}+S^{*}(\bar{z}) X_{*}^{+}: E_{*} \rightarrow E_{+}, & z \in \mathbb{C}_{-} \\
\Theta_{3}(z):=X^{+}+S^{*}(\bar{z}) X^{-}: E \rightarrow E_{+}, & z \in \mathbb{C}_{-} \\
\Theta_{4}(z):=X_{*}^{+}-S(z) X_{*}^{-}: E_{*} \rightarrow E_{-}, & z \in \mathbb{C}_{+}
\end{array}
$$

By virtue of (3.21) functions $\Theta_{j}, j=1,2,3,4$ are invertible for suitable values of $z \in \mathbb{C}_{ \pm}$and

$$
\begin{aligned}
& {\left[\Theta_{1}(z)\right]^{-1}=X^{-}+\Theta^{*}(\bar{z}) X_{*}^{+}, \quad z \in \rho(L) \cap \mathbb{C}_{+}} \\
& {\left[\Theta_{2}(z)\right]^{-1}=-X_{*}^{-}+\Theta(z) X^{+}, \quad z \in \rho\left(L^{*}\right) \cap \mathbb{C}_{-}} \\
& {\left[\Theta_{3}(z)\right]^{-1}=X^{+}+\Theta^{*}(\bar{z}) X_{*}^{-}, \quad z \in \rho(L) \cap \mathbb{C}_{-}} \\
& {\left[\Theta_{4}(z)\right]^{-1}=X_{*}^{+}-\Theta(z) X^{-}, \quad z \in \rho\left(L^{*}\right) \cap \mathbb{C}_{+}}
\end{aligned}
$$


Note as well that properties of projections $X^{ \pm}, X_{*}^{ \pm}$yield

$$
\begin{array}{lll}
X^{+}\left[\Theta_{1}(z)\right]^{-1}=X^{+} \Theta^{*}(\bar{z}) X_{*}^{+} & =\left[\Theta_{2}^{*}(\bar{z})\right]^{-1} X_{*}^{+}, \quad z \in \rho(L) \cap \mathbb{C}_{+} \\
X_{*}^{+}\left[\Theta_{2}(z)\right]^{-1}=X_{*}^{+} \Theta(z) X^{+} & =\left[\Theta_{1}^{*}(\bar{z})\right]^{-1} X^{+}, \quad z \in \rho\left(L^{*}\right) \cap \mathbb{C}_{-} \\
X^{-}\left[\Theta_{3}(z)\right]^{-1}=X^{-} \Theta^{*}(\bar{z}) X_{*}^{-} & =-\left[\Theta_{4}^{*}(\bar{z})\right]^{-1} X_{*}^{-}, \quad z \in \rho(L) \cap \mathbb{C}_{-} \\
X_{*}^{-}\left[\Theta_{4}(z)\right]^{-1}=-X_{*}^{-} \Theta(z) X^{-} & =-\left[\Theta_{3}^{*}(\bar{z})\right]^{-1} X^{-}, \quad z \in \rho\left(L^{*}\right) \cap \mathbb{C}_{+}
\end{array}
$$

Finally, we need to reformulate identities from Theorem 3.8 and Corollary 3.9 in terms of operators $A$ and $L$. To that end let us recall the definition of operators $\Gamma, \Gamma_{*}$ given in Section 2. Using the same arguments applied to the operator $L$ introduce two operators $G, G_{*}$ as closures of mappings $u \mapsto \frac{1}{\sqrt{2}} J \Delta_{V}(L+i I) u$, $v \mapsto \frac{1}{\sqrt{2}} J_{*} \Delta_{V^{*}}\left(L^{*}-i I\right) v$ initially defined on $u \in \operatorname{Dom}(L), v \in \operatorname{Dom}\left(L^{*}\right)$, respectively. Results of Theorem 3.8 and Corollary 3.9 now can be rewritten as identities

$$
\begin{array}{llll}
p^{\prime} \Gamma_{*}\left(A^{*}-z I\right)^{-1}(L-z I) u & =\Theta_{1}(z) J G u, & u \in \operatorname{Dom}(L), & z \in \mathbb{C}_{+} \\
p \Gamma(A-z I)^{-1}\left(L^{*}-z I\right) u & =\Theta_{2}(z) G_{*} u, & u \in \operatorname{Dom}\left(L^{*}\right), & z \in \mathbb{C}_{-} \\
p \Gamma(A-z I)^{-1}(L-z I) u & =\Theta_{3}(z) J G u, & u \in \operatorname{Dom}(L), & z \in \mathbb{C}_{-} \\
p^{\prime} \Gamma_{*}\left(A^{*}-z I\right)^{-1}(L-z I) u & =\Theta_{4}(z) G_{*} u, & u \in \operatorname{Dom}\left(L^{*}\right), & z \in \mathbb{C}_{+}
\end{array}
$$

Operator functions $\Theta_{j}, j=1,2,3,4$ on the right hand side of these formulae are boundedly invertible in accordance with (3.23).

\section{Functional model of a NON-Dissipative operator}

This Section is dedicated to the functional model of operator $L$ based on the symmetric form of Sz.-Nagy-Foiaş model for dissipative operator $A$ constructed in Section 2. To simplify considerations below, introduce the Hilbert space $\mathbf{H}_{L}$ obtained from the model space $\mathbf{H}=L_{2}\left(\begin{array}{cc}I & \mathcal{S}^{*} \\ \mathcal{S} & I\end{array}\right)$ by an isometry $\Pi: \mathbf{H} \rightarrow \mathbf{H}_{L}$ defined as the diagonal matrix $\operatorname{diag}\left(p, p^{\prime}\right)$ acting on elements of $\mathbf{H}$. Here $p$ and $p^{\prime}$ are isometries $p: E \rightarrow E_{+}, p: E_{*} \rightarrow E_{-}$from (3.12). Obviously, the space $\mathbf{H}_{L}$ is the model space for operator $A$ as well. The norm in $\mathbf{H}_{L}$ is analogous to (2.14)

$$
\left\|\left(\begin{array}{c}
\widetilde{g} \\
g
\end{array}\right)\right\|_{\mathbf{H}_{L}}^{2}:=\int_{\mathbb{R}}\left\langle\left(\begin{array}{cc}
I & S^{*} \\
S & I
\end{array}\right)\left(\begin{array}{l}
\widetilde{g} \\
g
\end{array}\right),\left(\begin{array}{l}
\widetilde{g} \\
g
\end{array}\right)\right\rangle_{E_{+} \oplus E_{-}} d k
$$

Model Theorem 2.8 holds true for mappings $(2.16)$ replaced by $p \mathscr{F}_{+}, p^{\prime} \mathscr{F}_{-}$, respectively, characteristic function $\mathcal{S}$ replaced by $S$, and the new spectral isometry $\Pi \Phi: \mathscr{H} \rightarrow \mathbf{H}_{L}$ that maps the dilation $\mathscr{A}$ into the multiplication operator on $\mathbf{H}_{L}$. In particular,

$$
\widetilde{g}+S^{*} g=p \mathscr{F}_{+} h, \quad S \widetilde{g}+g=p^{\prime} \mathscr{F}_{-} h,
$$

where $h \in H,(\widetilde{g}, g):=\Pi \Phi h$. Below the space $\mathbf{H}_{L}$ serves as the model space for the non-dissipative operator $L$. We denote $\mathcal{D}_{ \pm}:=\Pi \mathfrak{D}_{ \pm}, \mathcal{H}:=\Pi \mathfrak{H}, \mathcal{H}=\mathbf{H}_{L} \ominus\left[\mathcal{D}_{-} \oplus \mathcal{D}_{+}\right]$. Finally, let $P_{\mathcal{H}}$ be the orthogonal projection from $\mathbf{H}_{L}$ onto $\mathcal{H}$. 


\subsection{Model theorem.}

Theorem 4.1. In the model space $\mathbf{H}_{L}$ of dissipative operator $A$ the action of resolvents $(L-z I)^{-1},\left(L^{*}-z I\right)^{-1}$ are described by the following formulae with $(\widetilde{g}, g) \in \mathcal{H}, z \in \rho(L) \cap \mathbb{C}_{-}, \zeta \in \rho(L) \cap \mathbb{C}_{+}$

$$
\begin{aligned}
(L-z I)^{-1}\left(\begin{array}{l}
\widetilde{g} \\
g
\end{array}\right) & =P_{\mathcal{H}}(k-z)^{-1}\left(\begin{array}{c}
\widetilde{g} \\
g-X^{-}\left[\Theta_{3}(z)\right]^{-1}\left(\widetilde{g}+S^{*} g\right)(z)
\end{array}\right), \\
(L-\zeta I)^{-1}\left(\begin{array}{c}
\widetilde{g} \\
g
\end{array}\right) & =P_{\mathcal{H}}(k-\zeta)^{-1}\left(\begin{array}{c}
\widetilde{g}-X^{+}\left[\Theta_{1}(\zeta)\right]^{-1}(S \widetilde{g}+g)(\zeta) \\
g
\end{array}\right), \\
\left(L^{*}-\bar{\zeta} I\right)^{-1}\left(\begin{array}{c}
\widetilde{g} \\
g
\end{array}\right) & =P_{\mathcal{H}}(k-\bar{\zeta})^{-1}\left(\begin{array}{c} 
\\
g-X_{*}^{+}\left[\Theta_{2}(\bar{\zeta})\right]^{-1}\left(\widetilde{g}+S^{*} g\right)(\bar{\zeta})
\end{array}\right), \\
\left(L^{*}-\bar{z} I\right)^{-1}\left(\begin{array}{c}
\widetilde{g} \\
g
\end{array}\right) & =P_{\mathcal{H}}(k-\bar{z})^{-1}\left(\begin{array}{r}
\widetilde{g}+X_{*}^{-}\left[\Theta_{4}(\bar{z})\right]^{-1}(S \widetilde{g}+g)(\bar{z}) \\
g
\end{array}\right)
\end{aligned}
$$

Here symbol $\left(\widetilde{g}+S^{*} g\right)(z)$ denotes the value of analytic continuation of the vectorfunction $\widetilde{g}+S^{*} g \in H_{2}^{-}\left(E_{+}\right)$to the non-real point $z \in \mathbb{C}_{-}$. The symbol $(S \widetilde{g}+g)(z)$, $z \in \mathbb{C}_{+}$has an analogous meaning.

Note that the model equalities (2.13) for dissipative operator $A$ are the special case of Theorem 4.1. They are recovered if one assumes $J=I, J_{*}=I$, to so that projections $X^{-}$and $X_{*}^{-}$are the null operators. In this case, of course, $L$ and $A$ coincide.

4.2. Proof of Model theorem. In order to prove Theorem 4.1 we need two Lemmas.

Lemma 4.2. For $z \in \mathbb{C}_{+}, \zeta \in \mathbb{C}_{-}$and $z_{0} \in \rho(L)$

$$
\begin{aligned}
& p^{\prime} \Gamma_{*}\left(A^{*}-z I\right)^{-1}\left(L-z_{0} I\right)^{-1} \\
& =\left(z-z_{0}\right)^{-1}\left[p^{\prime} \Gamma_{*}\left(A^{*}-z I\right)^{-1}-\Theta_{1}(z) J G\left(L-z_{0} I\right)^{-1}\right], \\
& p \Gamma(A-\zeta I)^{-1}\left(L^{*}-\bar{z}_{0} I\right)^{-1} \\
& =\left(\zeta-\bar{z}_{0}\right)^{-1}\left[p \Gamma(A-\zeta I)^{-1}-\Theta_{2}(\zeta) G_{*}\left(L^{*}-\bar{z}_{0} I\right)^{-1}\right], \\
& p \Gamma(A-\zeta I)^{-1}\left(L-z_{0} I\right)^{-1} \\
& =\left(\zeta-z_{0}\right)^{-1}\left[p \Gamma(A-\zeta I)^{-1}-\Theta_{3}(\zeta) J G\left(L-z_{0} I\right)^{-1}\right], \\
& p^{\prime} \Gamma_{*}\left(A^{*}-z I\right)^{-1}\left(L^{*}-\bar{z}_{0} I\right)^{-1} \\
& =\left(z-\bar{z}_{0}\right)^{-1}\left[p^{\prime} \Gamma_{*}\left(A^{*}-z I\right)^{-1}-\Theta_{4}(z) G_{*}\left(L^{*}-\bar{z}_{0} I\right)^{-1}\right]
\end{aligned}
$$

Proof. Let $u=\left(L-z_{0} I\right)^{-1} h, h \in H$ in the first and third formulae of (3.24) and $u=\left(L^{*}-\bar{z}_{0} I\right)^{-1} h, h \in H$ in the second and fourth. The desired result is obtained by the equality $(L-z I)\left(L-z_{0} I\right)^{-1}=I+\left(z-z_{0}\right)\left(L-z_{0} I\right)^{-1}$.

The proof is complete. 
Lemma 4.3. For $h \in H,(\widetilde{g}, g):=\Pi \Phi$ and for almost all $k \in \mathbb{R}$ the identities hold:

For $z_{0} \in \rho(L) \cap \mathbb{C}_{-}$

$$
\begin{aligned}
& p \mathscr{F}_{+}\left(L-z_{0} I\right)^{-1} h=\left(k-z_{0}\right)^{-1}\left[\left(\widetilde{g}+S^{*} g\right)(k)-\Theta_{3}(k)\left[\Theta_{3}\left(z_{0}\right)\right]^{-1}\left(\widetilde{g}+S^{*} g\right)\left(z_{0}\right)\right] \\
& p^{\prime} \mathscr{F}_{-}\left(L-z_{0} I\right)^{-1} h=\left(k-z_{0}\right)^{-1}\left[(S \widetilde{g}+g)(k)-\Theta_{1}(k)\left[\Theta_{3}\left(z_{0}\right)\right]^{-1}\left(\widetilde{g}+S^{*} g\right)\left(z_{0}\right)\right] \\
& p \mathscr{F}_{+}\left(L^{*}-\bar{z}_{0} I\right)^{-1} h=\left(k-\bar{z}_{0}\right)^{-1}\left[\left(\widetilde{g}+S^{*} g\right)(k)-\Theta_{2}(k)\left[\Theta_{4}\left(\bar{z}_{0}\right)\right]^{-1}(S \widetilde{g}+g)\left(\bar{z}_{0}\right)\right] \\
& p \mathscr{F}_{-}\left(L^{*}-\bar{z}_{0} I\right)^{-1} h=\left(k-\bar{z}_{0}\right)^{-1}\left[(S \widetilde{g}+g)(k)-\Theta_{4}(k)\left[\Theta_{4}\left(\bar{z}_{0}\right)\right]^{-1}(S \widetilde{g}+g)\left(\bar{z}_{0}\right)\right]
\end{aligned}
$$

For $z_{0} \in \rho(L) \cap \mathbb{C}_{+}$

$$
\begin{aligned}
& p \mathscr{F}_{+}\left(L-z_{0} I\right)^{-1} h=\left(k-z_{0}\right)^{-1}\left[\left(\widetilde{g}+S^{*} g\right)(k)-\Theta_{3}(k)\left[\Theta_{1}\left(z_{0}\right)\right]^{-1}(S \widetilde{g}+g)\left(z_{0}\right)\right] \\
& p^{\prime} \mathscr{F}_{-}\left(L-z_{0} I\right)^{-1} h=\left(k-z_{0}\right)^{-1}\left[(S \widetilde{g}+g)(k)-\Theta_{1}(k)\left[\Theta_{1}\left(z_{0}\right)\right]^{-1}(S \widetilde{g}+g)\left(z_{0}\right)\right] \\
& p \mathscr{F}_{+}\left(L^{*}-\bar{z}_{0} I\right)^{-1} h=\left(k-\bar{z}_{0}\right)^{-1}\left[\left(\widetilde{g}+S^{*} g\right)(k)-\Theta_{2}(k)\left[\Theta_{2}\left(\bar{z}_{0}\right)\right]^{-1}\left(\widetilde{g}+S^{*} g\right)\left(\bar{z}_{0}\right)\right] \\
& p \mathscr{F}_{-}\left(L^{*}-\bar{z}_{0} I\right)^{-1} h=\left(k-\bar{z}_{0}\right)^{-1}\left[(S \widetilde{g}+g)(k)-\Theta_{4}(k)\left[\Theta_{2}\left(\bar{z}_{0}\right)\right]^{-1}\left(\widetilde{g}+S^{*} g\right)\left(\bar{z}_{0}\right)\right]
\end{aligned}
$$

Here the symbols like $\Theta_{1}(k), k \in \mathbb{R}$ stand for strong boundary values of the bounded analytic operator function $\Theta_{1}$ expressed via $S(k)$ and $S^{*}(k)$ according to (3.22). Vectors $(S \widetilde{g}+g)(\cdot)$ and $\left(\widetilde{g}+S^{*} g\right)(\cdot)$ are values of analytical continuations of respective vector-functions of Hardy classes to non-real points.

Proof. We only show how to verify the first equality. Others are proven similarly. Let $z_{0} \in \rho(L) \cap \mathbb{C}_{-}$. Lemma 4.2 and third identity in (3.24) yield for $h \in H$

$$
\begin{aligned}
p \Gamma(A-\zeta I)^{-1}\left(L-z_{0} I\right)^{-1} h & \\
& =\left(\zeta-z_{0}\right)^{-1}\left[p \Gamma(A-\zeta I)^{-1} h-\Theta_{3}(\zeta)\left[\Theta_{3}\left(z_{0}\right)\right]^{-1} p \Gamma\left(A-z_{0} I\right)^{-1} h\right]
\end{aligned}
$$

Put $\zeta=k-i \varepsilon, k \in \mathbb{R}, \varepsilon>0$. Then as $\varepsilon \downarrow 0$ we obtain the required identity for almost all $k \in \mathbb{R}$ by virtue of (4.1) and definitions (2.16).

Now we are ready to prove Theorem 4.1.

Proof of Model Theorem 4.1. Let us consider the first equality. Denote $\left(\tilde{f}_{z}, f_{z}\right)$ the vector on the right hand side. It is suffice to verify that for $z \in \rho(L) \cap \mathbb{C}_{-}$and $h \in H$ almost everywhere on the real axis

$$
\widetilde{f}_{z}+S^{*} f_{z}=p \mathcal{F}_{+}(L-z I)^{-1} h, \quad \widetilde{S} f_{z}+f_{z}=p^{\prime} \mathcal{F}_{-}(L-z I)^{-1} h,
$$

where $h \in H$ is related to the vector $(\widetilde{g}, g)$ as $(\widetilde{g}, g)=\Pi \Phi h$. Direct computation according to (2.15) yields

$$
\left(\begin{array}{c}
\widetilde{f}_{z} \\
f_{z}
\end{array}\right)=\frac{1}{k-z}\left(\begin{array}{l}
\widetilde{g}-X^{+}\left[\Theta_{3}(z)\right]^{-1}\left(\widetilde{g}+S^{*} g\right)(z) \\
g-X^{-}\left[\Theta_{3}(z)\right]^{-1}\left(\widetilde{g}+S^{*} g\right)(z)
\end{array}\right)
$$

therefore

$$
\begin{aligned}
\widetilde{f}_{z}+S^{*} f_{z} & =(k-z)^{-1}\left[\widetilde{g}+S^{*} g-\left(X^{+}+S^{*} X^{-}\right)\left[\Theta_{3}(z)\right]^{-1}\left(\widetilde{g}+S^{*} g\right)(z)\right] \\
& =(k-z)^{-1}\left[\left(\widetilde{g}+S^{*} g\right)(k)-\Theta_{3}(k)\left[\Theta_{3}(z)\right]^{-1}\left(\widetilde{g}+S^{*} g\right)(z)\right]
\end{aligned}
$$


which coincides with $\left(p \mathscr{F}_{+}(L-z I)^{-1} h\right)(k)$ by Lemma 4.3. Thus, first equality (4.2) is obtained. Analogously, for almost all $k \in \mathbb{R}$

$$
\begin{aligned}
S \widetilde{f}_{z}+f_{z} & =(k-z)^{-1}\left[S \widetilde{g}+S^{*} g-\left(S X^{+}+X^{-}\right)\left[\Theta_{3}(z)\right]^{-1}\left(\widetilde{g}+S^{*} g\right)(z)\right] \\
& =(k-z)^{-1}\left[\left(S \widetilde{g}+S^{*} g\right)(k)-\Theta_{1}(k)\left[\Theta_{3}(z)\right]^{-1}\left(\widetilde{g}+S^{*} g\right)(z)\right] \\
& =\left(p^{\prime} \mathscr{F}_{-}(L-z I)^{-1} h\right)(k) .
\end{aligned}
$$

Hence, equalities (4.2) are validated. Other statements of Theorem are proven similarly.

The proof is complete.

\section{REFERENCES}

[1] B.P. Allahverdiev, On dilation theory and spectral analysis of dissipative Schrödinger operators in Weyl's limit-circle case (Russian), Izv. Akad. Nauk. SSSR Ser. Mat. 54 (1990), 242-257; English transl.: Math. USSR Izv. 36 (1991), 247-262.

[2] B.P. Allahverdiev, Extensions, Dilation and Functional Models of Dirac Operators, Integr. Equ. Oper. Theory 51 (2005), 459-475

[3] B.P. Allahverdiev, Extensions, Dilation and Functional Models of Discrete Dirac Operators, Illinois J. of Math. 47, 3 (2003), 831-845

[4] B.P. Allahverdiev, Dilation and Functional Model of Dissipative Operator Generated by an Infinite Jacobi Matrix, Math. and Comp. Modelling 38, 3 (2003), 989-1001.

[5] D.Z Arov, Passive linear stationary dynamic systems. (Russian) Sibirsk. Mat. Zh. 20, 2 (1979), 211-228.; English Transl.: Siberian Mathematical Journal, 20 (2), 149-162.

[6] T.Ya. Azizov, I.S. Iokhvidov, Linear Operators in Spaces With an Indefinite Metric, John Wiley \& Sons Inc., 1989.

[7] J. Ball, Models for Noncontractions, J. Math. Anal. Appl., 52 (1975), 235-254

[8] M. Baro, H. Neidhardt Dissipative Schrödinger-type operators as a model for generation and recombination, J. Math. Phys. 44, 6 (2003), 2373-2401.

[9] M. Baro, H.-Chr. Kaiser, H. Neidhardt, J. Rehberg, Dissipative Schrödinger-Poisson systems, J. Math. Phys. 45, 1 (2004), 21-43.

[10] L. de Branges, J. Rovnyak, Canonical models in quantum scattering theory, in "Perturbation Theory and its Applications in Quantum Mechanics" (C. H. Wilcox, Ed.), Wiley, New York, 1966.

[11] D. Clark, On models for noncontracfions, Acta Sci. Math., 36, 5-6 (1974).

[12] H. Dym, H. McKean, Gaussian Processes, Function Theory, and the Inverse Spectral Problem, Academic Press, 1976.

[13] B. McEnnis, Models for operators with bounded characteristic function. Acta Sci. Math. (Szeged) 43 (1981), no. 1-2, 71-90.

[14] P. Fuhrmann, Linear Systems and Operators in Hilbert Space, MGraw-Hill, New York, 1981.

[15] H. Goedbloed S. Poedts, Principles of Magnetohydrodynamics: With Applications to Laboratory and Astrophysical Plasmas, Cambridge University Press, 2004.

[16] I. S. Iohvidov, A certain class of linear-fractional operator transformations. (Russian) A collection of articles on function spaces and operator equations (Proc. Sem. Functional Anal., Math. Fac., Voronezh State Univ., Voronezh, 1970) (Russian), pp. 18-44. Voronež. Gos. Univ., Voronezh, 1970.

[17] H.-Ch. Kaiser, H. Neidhardt, J. Rehberg, On 1-dimensional dissipative Schrödinger-type operators, their dilations, and eigenfunction expansions. Math. Nachr. 252 (2003), 51-69.

[18] V. V. Kapustin, Spectral analysis of almost unitary operators, Algebra i Analiz 13 (2001), no.5, 44-68; English transl.: St.Petersburg Math.J. 13 (2002), no.5, 739-756.

[19] Yu. Kudryashov, Symmetric and selfadjoint dilations of dissipative operators. (Russian) Teor. Funktsiǔ, Funktsional. Anal. i Prilozhen. 37 (1982), 51-54.

[20] A.V. Kiselev Functional Model for Singular Perturbations of Non-self-adjoint Operators, in Operator Theory: Advances and Applications, Vol. 174, (2007), Birkäuser, 99-115.

[21] A.V. Kiselev, S.N. Naboko Nonself-Adjoint Operators with Almost Hermitian Spectrum: : Matrix model. I, J. Comp. and Appl. Math. 194, 1, 2006 p. 115-130. 
[22] A.V. Kiselev, S.N. Naboko Nonself-Adjoint Operators with Almost Hermitian Spectrum: Weak Annihilators Funct. Anal. and Its Appl., 38, 3, 2004, pp. 192-201.

[23] Yu.A. Kuperin, S.N. Naboko, R.V. Romanov, Spectral analysis of transport operator: Functional model approach, Indiana Univ. Math. J. 51, 6 (2002), 13891426.

[24] P. Lax, R. Phillips, Scattering theory. Pure and Applied Mathematics, Vol. 26 Academic Press, New York-London, 1967.

[25] N.G. Makarov, V.I. Vasyunin, A model for noncontractions and stability of the continuous spectrum. Lect.Notes in Math., 864 (1981), 365-412.

[26] S.N. Naboko, Absolutely continuous spectrum of a nondissipative operator, and a functional model. I. (Russian) Investigations on linear operators and the theory of functions, VII. Zap. Naučn. Sem. Leningrad. Otdel Mat. Inst. Steklov. (LOMI) 65 (1976), 90-102. English transl.: J. Sov. Math. 16, 3 (1981)

[27] S.N. Naboko, Absolutely continuous spectrum of a nondissipative operator, and a functional model. II. (Russian) Investigations on linear operators and the theory of functions, VIII. Zap. Nauchn. Sem. Leningrad. Otdel. Mat. Inst. Steklov. (LOMI) 73 (1977). English transl.: J. Math. Sci. 34, 6 (1986), 2090-2101.

[28] S.N. Naboko, Functional model of perturbation theory and its applications to scattering theory. (Russian) Boundary value problems of mathematical physics, 10. Trudy Mat. Inst. Steklov. 147 (1980), 86-114.

[29] S.N. Naboko, Conditions for the existence of wave operators in the nonselfadjoint case. (Russian) Wave propagation. Scattering theory, Probl. Mat. Fiz., 12, Leningrad. Univ., Leningrad, $1987,132-155$

[30] S.N. Naboko, R.V. Romanov, Spectral singularities, Szökefalvi-Nagy-Foias functional model and the spectral analysis of the Boltzmann operator. Recent advances in operator theory and related topics (Szeged, 1999), 473-490, Oper. Theory Adv. Appl., 127, Birkhäuser, Basel, 2001.

[31] S.N. Naboko, V.F. Veselov, The determinant of the characteristic function and the singular spectrum of a nonselfadjoint operator. (Russian) Mat. Sb. (N.S.) 129(171) (1986), no. 1, 20-39.

[32] B. Szökefalvi-Nagy, C. Foiaş, Harmonic Analysis of Operators on Hilbert Space, North Holland, New York, 1970.

[33] N.K. Nikolskiú, Treatise on the Shift Operator, Springer-Verlag, Berlin etc., 1986.

[34] N.K. Nikolskiu,, Operators, Functions, and Systems: An Easy reading, v.2: Model Operators and Systems, AMS, 2002.

[35] B.S. Pavlov, On conditions for separation of the spectral components of a dissipative operator, Izv. Akad. Nauk SSSR, Ser. Matem., 39 (1975), 123-148 (Russian); English transl.: Math USSR Izvestiya, 9 (1976), 113-137.

[36] B.S. Pavlov, Selfadjoint dilation of a dissipative Schrödinger operator and expansion in its eigenfunction. (Russian) Mat. Sb. (N.S.) 102 (144), 4 (1977), 511-536.

[37] B.S. Pavlov, Dilation theory and spectral analysis of nonselfadjoint differential operators, Proc. 7th Winter School, Drobobych 1974, 3-69 (1976) (Russian); English transl: Transl. II. Ser., Am. Math. Soc. 115 (1981), 103-142.

[38] B.S. Pavlov, Spectral Analysis of a dissipative singular Schrödinger operator in terms of a fucntional model, in M. A. Schubin (Ed.) Partial Differential Equations VIII, Encyclopaedia Math Sci. Vol. 65, Springer, Berlin, 1996, pp. 87-153.

[39] B.S. Pavlov, Irreversibility, Lax-Phillips approach to resonance scattering and spectral analysis of non-selfadjoint operators in Hilbert space, Int. J. Theor. Phys. 38, No.1, 21-45, 1999.

[40] B.S. Pavlov, A Remark on Spectral Meaning of the Symmetric Functional Model, in Operator Theory: Advances and Applications, Vol. 154, Birkhäuser Verlag, Basel/Schiwtzerland, 2004, 163-177.

[41] M. Rosenblum, J. Rovnyak, Hardy calsses and operator theory, Oxford University Press, 1985.

[42] V. A. Ryzhov, Absolutely continuous and singular subspaces of a nonselfadjoint operator. (Russian) Zap. Nauchn. Sem. S.-Peterburg. Otdel. Mat. Inst. Steklov. (POMI) 222 (1995), Issled. po Linein. Oper. i Teor. Funktsii. 23, 163-202; translation in J. Math. Sci. (New York) 87 (1997), no. 5, 3886-3911 
[43] V. A. Ryzhov, Rigged absolutely continuous subspaces and the stationary construction of wave operators in nonselfadjoint scattering theory. (Russian) Zap. Nauchn. Sem. S.-Peterburg. Otdel. Mat. Inst. Steklov. (POMI) 217 (1994), Issled. po Linein. Oper. i Teor. Funktsii. 22, 144-171; translation in J. Math. Sci. (New York) 85 (1997), no. 2, 1849-1866

[44] V. A. Ryzhov, Functional Model of a Class of Nonselfadjoint Extensions of Symmetric Operators, in Operator Theory: Advances and Applications, Vol. 174, (2007), Birkäuser, 117-158.

[45] V. A. Ryzhov, A General Boundary Value Problem and its Weyl Function. Submitted to Opuscula Math. 2007.

[46] Yu. L. Shmul'yan, The theory of linear relations and spaces with an indefinite metric Funct. Anal. and its Appl. Vol 10, 1 (1976) pp. 56-60.

[47] A.V. Štraus, Characteristic functions of linear operators. (Russian) Izv. Akad. Nauk. SSSR, Ser. Mat., 24, 1 (1960), 43-74; English transl. in AMS Transl. (2) 40 (1964), 1-37.

[48] A. S. Tikhonov, On a fucntional model of the K-relation, (Russian) Dinamicheskie Sistemy, No. 5, 1986, p. 106-111. Translated in J. Soviet Math. 65 (1993), no. 1, 1471-1474.

[49] A. S. Tikhonov, A functional model and duality of spectral components for operators with a continuous spectrum on a curve, Algebra i Analiz 14 (2002), no.4, 158-195; English transl.in: St.Petersburg Math.J. 14 (2003), no.4.

[50] D. Xia, On the analytic model of a class of hyponormal operators. Integral Equations Operator Theory 6 (1983), no. 1, 134-157.

[51] D. Yakubovich, Linearly similar model of Sz.-Nagy - Foias type in a domain (in Russian), Algebra i Analiz, 15, No. 2 (2003), 180-227, English transl. in St. Petersburg Math. J. 15, No. 2 (2004), 289-321.

[52] D. Yakubovich, Nagy-Foiaş type Funtinal Model of Nondissipative Operators in Non-Convex Parabolic Domains, Preprint arXiv:math.FA/0607062 v1, 3 July 2006, 31 p.

Suite 200 - 1628, Dickson Ave., Kelowna, BC, V1Y 9X1, Canada

E-mail address: vryzhov@bridges.com 